\title{
Medienethik: Vier populäre Irrtümer
}

\author{
Daniel Eggers
}

Eingegangen: 6. Februar 2021 / Angenommen: 2. Juni 2021 / Online publiziert: 1. Juli 2021

(C) Der/die Autor(en) 2021

Zusammenfassung Obwohl die Bedeutung medienethischer Reflexion und der Status der Medienethik als einer angewandten und damit normativen Ethik weithin anerkannt sind, bleiben die vorliegenden Beiträge oft unbefriedigend: Sie liefern kaum Antworten auf konkrete moralische Probleme, enthalten aber auch nur selten Theorieangebote, die das Potenzial hätten, derartige Antworten zu generieren. Ziel des Aufsatzes ist es, anhand von vier Beispielen theoretische Engführungen und Fehlstellungen der gegenwärtigen Debatte zu beschreiben, die einer fruchtbaren multidisziplinären Diskussion medienethischer Fragestellungen und einer genuin normativen Medienethik im Wege stehen.

Schlüsselwörter Angewandte Ethik · Moralische Begründung · Prinzipilismus · Verantwortung $\cdot$ Pflicht $\cdot$ Praxisnormen $\cdot$ Individualethik

\section{Media ethics: Four popular misconceptions}

Abstract Media ethics is concerned with the moral aspects of mass communication which is now conducted not only via traditional mass media such as television, radio and newspapers but also, and to an increasing degree, via the internet and social media. That these forms of media-related communication require ethical reflection is uncontroversial: Mass media communication gives rise to specific moral challenges for which there are no obvious solutions, given that they are typically characterized by conflicts of more general moral norms or values.

Although these facts are widely acknowledged, however, media ethics falls short of being a flourishing branch of the sciences, and this applies to German mediaethical discourse in particular. Moreover, the existing contributions often remain

Prof. Dr. Daniel Eggers $(\bowtie)$

Institut für Philosophie, Universität Regensburg, 93040 Regensburg, Deutschland

E-Mail: daniel.eggers@ur.de 
unsatisfactory: They rarely offer determinate answers to specific moral problems, nor do they provide theoretical approaches by means of which such answers can be generated. In virtue of this, media ethics runs the risk of becoming unfaithful to its own designation as a form of applied normative ethics.

The purpose of this paper is to inquire into possible reasons for this current state of affairs and to describe obstacles which prevent a more fruitful interdisciplinary discussion of media-ethical issues. To this end, I will critically address four fundamental assumptions which are widely shared in the relevant German literature. These are

(a) a general skepticism regarding the rational justification of moral norms and judgements,

(b) the idea that media ethics should not be aimed at the identification of "ideal" moral norms but ought rather to focus on the formulation of "practice norms" which explicitly take current media structures and the everyday practice of media agents into account,

(c) the view that the concept of responsibility is the key concept of media ethics and that this concept asks for a consequentialist approach, and

(d) the claim that, in virtue of the underlying organizational structures, modern media-related conduct cannot adequately be captured by individualist approaches but requires conceptions of collective action and responsibility.

The main problem with the first view consists in the transition from the idea that there can be no rational justification of fundamental or first ethical principles to the idea that any form of rational intersubjective moral justification is impossible. As a result, the potential of coherentist approaches to ethical justification has long been overlooked by media ethicists, although such approaches have not only dominated much of moral and political philosophy since the 1970s but also many debates in applied ethics.

The view according to which media-ethical discussion must not rest with abstract and ideal conceptions of moral media-related behaviour but needs to come up with "practice norms" requires revision insofar as the legitimate request for practical application is often confused with more far-reaching demands which would make media-ethical reflection subject to excessive constraints and deprive a more radical media critique of its basis.

The claim that, given the central importance of the concept of responsibility, media ethics must be conceived of in consequentialist terms, is based on an inadequate understanding of the concept of moral responsibility and the way in which it relates to the concept of a moral duty. It overlooks the possible role of "imperfect" duties as well as the fact that any reasonable attribution of moral responsibility must, at some point, lead into the formulation of individual obligations to act in one way rather than the other. Moreover, it derives from an inappropriate distinction of consequentalism and deontology which is often informed by Max Weber's distinction of "Gesinnungsethik" and "Verantwortungsethik".

Finally, the view that modern media-related behaviour can only be captured in collectivist terms underestimates the possible contribution of individualist approaches as well as the theoretical costs that attach to conceptions of collective action and collective responsibility. In order to be empirically and normatively adequate, media- 
ethical reflection is dependent on both individualist concepts and systemic analyses. What is needed, therefore, is an open and unbiased discussion of the strengths and weaknesses of both approaches and the concerted attempt to tell media-ethical challenges that require an answer in individualist terms from those which require an answer in collectivist terms.

Keywords Applied ethics · Moral justification · Principilism · Responsibility · Duty $\cdot$ Practice norms $\cdot$ Individualist ethics

\section{Einleitung: Medienethik als Problemfall angewandter Ethik}

Die Medienethik befasst sich mit den moralischen Aspekten der Massenkommunikation, wie sie sich mit Hilfe der traditionellen Massenmedien Fernsehen, Radio und Zeitung, in immer größerem Maß aber auch über das Internet und die sozialen Medien vollzieht. Dass diese medial vermittelten Formen der Kommunikation spezifische moralische Fragen aufwerfen und nach ethischer Orientierung verlangen, kann als unstrittig gelten. Von den unzähligen Beispielen, an denen sich die Notwendigkeit medienethischer Betrachtung illustrieren ließe, seien hier zwei ausgewählt:

Der Hanns-Joachim-Friedrichs-Preis für Fernsehjournalismus wird seit 1995 unter einem Motto vergeben, das auf Aussagen seines Namensgebers zurückgeht und sinnbildlich für das Ideal journalistischer Neutralität steht: „Einen guten Journalisten erkennt man daran, dass er sich nicht gemein macht mit einer Sache, auch nicht mit einer guten Sache." Obwohl das Zitat für viele zum Ausdruck bringt, was guten und seriösen Journalismus ausmacht, stellt die darin geforderte Art journalistischer Neutralität in der Praxis heute eher die Ausnahme dar. So sind viele aktuelle journalistische Beiträge etwa durch ein sichtbares Bekenntnis zu demokratischen Grundwerten und eine explizite Opposition zu Rassismus, Sexismus und anderen Formen der Diskriminierung gekennzeichnet. Dabei werden nicht nur diese allgemeineren Werte und Prinzipien vorausgesetzt oder verteidigt, sondern oft auch sehr spezifische Auffassungen darüber, was sie mit Blick auf konkrete Handlungszusammenhänge erfordern.

Nun ist keineswegs offensichtlich, dass diese Form von journalistischer Parteinahme falsch sein muss, so wie umgekehrt das von Friedrichs zum Ausdruck gebrachte journalistische Ethos nicht ohne Probleme ist. Dies zeigt auch die Debatte um Gegenwart und Zukunft des Journalismus, die im Juni 2020 im Spiegel geführt wurde und sich an der Entlassung des Meinungschefs der New York Times, James Bennett, entzündete. Während USA-Korrespondent Philipp Oehmke (2020) unter dem Titel „Die Zeit der Neutralität ist vorbei“ einen Abgesang auf das Ethos journalistischer Neutralität anstimmte, bemühte sich Redakteur Florian Gathmann (2020) um eine Verteidigung des Ideals, und zwar unter ausdrücklicher Bezugnahme auf Friedrichs. Gathmanns Beitrag zeigt dabei nicht nur, dass man selbst in Zeiten eines Donald Trump unterschiedlicher Meinung über den Wert und die Notwendigkeit journalistischer Neutralität sein kann. Er zeigt auch, dass erst noch zu bestimmen ist, was genau das Neutralitätsgebot mit Blick auf konkretes journalistisches Handeln impliziert. 
Die ethischen Herausforderungen, die sich im Kontext massenmedialen Handelns stellen, beschränken sich freilich nicht auf den Bereich des klassischen Journalismus und der politischen Berichterstattung. Ein Beispiel aus dem Bereich der Unterhaltung liefert die regelmäßig wiederkehrende Diskussion darüber, wie im Rahmen von Reality-Shows wie „Bauer sucht Frau“, „Schwiegertochter gesucht“ oder „Deutschland sucht den Superstar" mit den Kandidat*innen umgegangen wird. Ein häufig erhobener Vorwurf lautet, die Teilnehmer*innen und ihr Traum von Bekanntheit, Erfolg oder Liebe würden ausgenutzt, um möglichst hohe Quoten zu erzielen, sie würden ,,vorgeführt“, der Lächerlichkeit preisgegeben und damit für ihr weiteres Leben beschädigt (vgl. hierzu auch Mikos 2010, S. 432; Krämer 2020, S. 16-17).

Auch hier ist die Frage der angemessenen moralischen Bewertung keineswegs trivial, hängt ihre Beantwortung doch nicht zuletzt davon ab, wie man allgemeinere Werte wie Autonomie, Eigenverantwortung und Solidarität ausbuchstabiert und gewichtet. Muss man Personen, die an den betreffenden Formaten teilnehmen, weil sie bekannt oder berühmt werden wollen (und die dies ja in gewisser Weise auch werden), vor sich selbst schützen? Würde man sich damit nicht umgekehrt eines fragwürdigen Paternalismus schuldig machen und Menschen einer wichtigen Form der Teilhabe an den Massenmedien und gegebenenfalls sogar eines Instruments der Selbstermächtigung berauben?

Die beiden Beispiele zeigen, dass die vielfältigen Arten massenmedialen Handelns einen beträchtlichen Bedarf an ethischer Reflexion produzieren. Sie werfen moralische Probleme auf, deren gesellschaftliche Relevanz klar erkennbar ist, für die aber keine offensichtliche Lösung bereitsteht, weil sie typischerweise durch Konflikte von allgemeineren Werten oder Prinzipien gekennzeichnet sind, die auf unterschiedliche Weise bzw. in unterschiedliche Richtungen aufgelöst werden können.

Es ist daher wenig erstaunlich, dass es aktuell einen lebhaften öffentlichen Diskurs über Fragen der Medienmoral gibt, zumindest nicht, sofern man in Rechnung stellt, wie sehr sich die Teilhabe an der medialen Öffentlichkeit im Zuge der Digitalisierung und der sozialen Medien verändert hat. So vergeht gegenwärtig kaum eine Woche, in der nicht irgendein shitstorm Anlass zu einer Debatte über Medienverhalten und in der Folge zu öffentlichen Entschuldigungen, Rücktritten oder Absagen von Preisverleihungen und Veranstaltungen gibt.

Obwohl Geschwindigkeit und Wucht solcher Reaktionen erkennbar zugenommen haben, lässt sich die Auffassung vertreten, dass es sich nur um einen spezifisch modernen Umgang mit Medienskandalen handelt, wie es sie mit der Berichterstattung zur Barschel-Affäre, dem Gladbecker Geiseldrama oder dem Fall Sebnitz auch früher gegeben hat (vgl. dazu Schicha 2010a). Unterschiede bestehen vor allem darin, dass sich die öffentliche Artikulation moralischer Empörung heute in kürzer werdenden Abständen vollzieht und dass dabei nicht nur professionelle Medienschaffende, sondern zunehmend auch individuelle Mediennutzer*innen ins Kreuzfeuer geraten.

Selbst wenn die moralische Kritik medialen Verhaltens auf diese Weise aber zu einem permanenten Bestandteil öffentlicher Kommunikation geworden sein mag: Es wäre falsch, den alltäglichen Diskurs über Medien und Moral mit einer wissenschaftlich-systematischen Medienethik gleichzusetzen oder zu glauben, dass es einer solchen nicht mehr bedarf. Die über Onlinekommentare, Internetforen und soziale 
Medien artikulierte öffentliche Medienkritik ist nicht konsequent prinzipiengeleitet, sondern besteht zu einem Gutteil aus spontanen, mitunter widersprüchlichen emotionalen Reaktionen. Eine mit wissenschaftlichem Anspruch auftretende systematischanalytische Reflexion medialen Handelns kann sie nicht ersetzen. Zudem steht sie ihrerseits in der Kritik, wird sie doch oftmals als ,moralisierend“ und als Ausdruck einer „Empörungskultur“ abgewertet. Hie und da wird auch im Sinne des virtue signalling unterstellt, es gehe den Betreffenden gar nicht darum, aufrichtige moralische Urteile oder Werte zu kommunizieren, sondern sie wollten sich lediglich selbst als tugendhaft inszenieren, und das heißt hier: als jemanden, der auf der Seite der moralischen Mehrheit steht (vgl. Bartholomew 2015; für eine philosophische Verteidigung von virtue signalling siehe Levy 2020). Die Entschuldigungen, mit denen die jeweils Kritisierten auf die öffentliche Kritik reagieren, sehen sich mit ähnlichen Vorwürfen konfrontiert.

Gerade weil Moral und Moralisierung also in der Öffentlichkeit einen zunehmend großen Raum einnehmen und dabei nicht nur entgegengesetzte Positionen vertreten werden, sondern auch kontinuierlich moralische Unaufrichtigkeit unterstellt wird, braucht es eine wissenschaftliche Medienethik, die die ethischen Herausforderungen medialen Handelns systematisch reflektiert und gezielt der Frage nachgeht, welche der im öffentlichen Diskurs vorgebrachten Überzeugungen sich in rationaler Weise und im Sinne eines genuin moralischen Standpunktes begründen lassen (vgl. hierzu auch Eggers 2019).

Wer sich mit dieser Erwartung der medienethischen Literatur nähert, erfährt aber eine Enttäuschung. Obwohl Notwendigkeit und Bedeutung medienethischer Reflexion weithin anerkannt werden, ist die Medienethik insbesondere im deutschsprachigen Raum weit davon entfernt, ein fest etablierter und florierender Wissenschaftszweig zu sein. Diese Tatsache wird von vielen Medienethiker*innen auch recht freimütig eingestanden (vgl. etwa Funiok 2002, S. 37; 2011, S. 24; Brosda und Schicha 2010, S. 9-10; Thies 2011, S. 206; Filipovic 2015, S. 436; Prinzing et al. 2015, S. 9-10). Neben unzureichender theoretischer Fundierung und interdisziplinärer Ausrichtung wird dabei eine unzureichende normative Konkretisierung in Form spezifischer Handlungsanweisungen und Bewertungen bemängelt (vgl. Greis 2003, S. 3; Friedrichsen und Gertler 2011, S. 85; Krainer 2015, S. 48-49; Weber 2016, S. 58; Zurstiege 2018, S. 131; Altmeppen et al. 2019, S. 71-72; Krainer und Karmasin 2020, S. 312).

Ein Zugeständnis ist an dieser Stelle gleichwohl angebracht: Es gibt in der deutschsprachigen Wissenschaftslandschaft erkennbar Bemühungen, die wissenschaftliche Diskussion medienethischer Fragestellungen zu befördern und dabei einen interdisziplinären Austausch voranzutreiben. Diese Bemühungen, die bis zur Gründung des Netzwerks Medienethik im Jahr 1997 und der Fachgruppe Kommunikations- und Medienethik der Deutschen Gesellschaft für Publizistik und Kommunikationswissenschaft im Jahr 2001 zurückreichen, haben gerade in den letzten Jahren mit der Etablierung der Schriftenreihe „Kommunikations- und Medienethik“ beim Nomos Verlag und der Neuausrichtung der Zeitschrift Communicatio Socialis sowie der Einrichtung von Lehrstühlen für Medienethik in München und Erlangen-Nürnberg an Fahrt aufgenommen (vgl. hierzu Altmeppen et al. 2013, S. 280-81; Filipovic et al. 2017, S. 3). So sehr diese Bestrebungen aber Anlass zur 
Hoffnung geben mögen, bleiben sie bislang auf einen überschaubaren Personenkreis beschränkt, und einige der Probleme, welche die ältere medienethische Literatur prägen, kennzeichnen auch die neueren Veröffentlichungen, die aus den genannten Aktivitäten hervorgegangen sind.

So lässt sich der Großteil medienethischer Veröffentlichungen einer der drei folgenden Kategorien zuordnen:

a. deskriptiv-empirisch angelegte Beiträge, vornehmlich aus dem Bereich der Kommunikationswissenschaften, in denen beispielsweise die Wertüberzeugungen von Medienschaffenden und deren Entstehung erforscht, medienethische Selbstverpflichtungskodizes analysiert oder Aktivitäten untersucht werden, mit denen Medienunternehmen ihrer ethischen Verantwortung gerecht zu werden versuchen, in denen aber keine eigene ethische Bewertung erfolgt (vgl. z. B. Bracker 2017; Betz et al. 2018; Keel 2018; Keel et al. 2018; Lauerer 2018; Müller et al. 2018; Altendorfer 2019; Koinig et al. 2019; Wiedel 2019; Wladarsch 2019; Kaiser und Zeilinger 2021; Kelm et al. 2021; Sell und Oswald 2021). ${ }^{1}$

b. Beiträge, in denen zwar konkrete Bewertungen vorgenommen und Handlungsanweisungen formuliert oder zumindest angedeutet werden, in denen dies aber nicht auf Grundlage einer systematisch entwickelten normativen Theorie geschieht (vgl. z. B. Dernbach 2017; Jackob 2018). ${ }^{2}$

c. Beiträge, wie sie sich vornehmlich in den vielen Handbüchern und Sammelbänden finden, welche die deutschsprachige Literatur nach wie vor dominieren (vgl. hierzu auch Funiok 2015, S. 20-21; Krainer 2015, S. 35), in denen medienethische Herausforderungen und Probleme identifiziert werden, die eine eingehendere wissenschaftlich-normative Betrachtung verdienen, wobei diese Betrachtung aber ein Versprechen auf die Zukunft bleibt (vgl. z. B. Debatin 2015; Ring und Funiok 2015; Gossel 2018; Leif 2018; Liesem 2018; Porlezza 2020; Stapf 2020; Krainer und Karmasin 2020; Schicha 2021).

Angesichts dieser Ausrichtung der vorliegenden Beiträge kann die Medienethik letztlich ihrem selbst gesetzten Ziel nicht gerecht werden. Die Medienethik ist, wie auch die Bio- oder Medizinethik, die Umweltethik oder die Wissenschaftsethik, eine Form der angewandten Ethik (vgl. auch Teichert 1996, S. 809-10; Wiegerling 1998, S. 5; Wunden 1999, S. 37; Funiok 2002, S. 41; 2011, S. 24; Rath 2002, S. 62; 2010, S. 143; Krainer 2002, S. 156; Greis et al. 2003, S. xi; Stapf 2006, S. 16; Alsdorf 2007, S. 24-25; Bayertz 2008, S. 166; Brosda und Schicha 2010, S. 11-12; Friedrichsen und Gertler 2011, S. 85; Thies 2011, S. 206; Maring 2014, S. 10;

\footnotetext{
1 Zur empiristischen Ausrichtung der kommunikationswissenschaftlichen Medienethik vgl. auch Altmeppen et al. (2013, S. 280), und Zurstiege (2018, S. 130).

2 Entweder wird in diesen Fällen auf eine theoretische Fundierung gänzlich verzichtet, oder Bewertungen und Handlungsanweisungen werden in eklektischer und nicht ganz nachvollziehbarer Weise aus einem allgemeinen Fundus ethischer Theorien ,abgeleitet“, der typischerweise die aristotelische Tugendethik, den Utilitarismus, Kants Lehre vom kategorischen Imperativ und die Diskursethik umfasst. Weitere Beispiele hierfür finden sich vor allem in der englischsprachigen Literatur, in der es zahlreiche Handbücher zur Medienethik gibt, in denen anhand von Fallbeispielen konkrete Handlungsanweisungen für professionelle Handlungskontexte entwickelt werden. Vgl. etwa Black und Roberts (2011); Frost (2016); Bobbitt (2020) sowie die Beiträge in Maisel und Dineen (2018); Fox und Saunders (2019); Luce (2019).
} 
Karmasin und Litschka 2014, S. 367; Köberer 2015, S. 99; Filipovic 2016, S. 41; Zurstiege 2018, S. 129-30). Als solche gehört sie in den übergeordneten Kontext der normativen Ethik. Entsprechend besteht ihre Aufgabe darin, Kriterien für die Bewertung medialen Handelns zu entwickeln und konkrete Handlungsweisungen für die massenmediale Praxis zu formulieren (zur normativen Ausrichtung der Medienethik bzw. der angewandten Ethik allgemein vgl. auch Wunden 1999, S. 38; Funiok 2002, S. 38; Stapf 2006, S. 16, 24; Bayertz 2008, S. 173; Heesen 2008, S. 270; Brosda und Schicha 2010, S. 12; Friedrichsen und Gertler 2011, S. 71; Thies 2011, S. 206; Altmeppen et al. 2013, S. 281; Maring 2014, S. 14; Karmasin und Litschka 2014, S. 375; Köberer 2015, S. 110-11; Zurstiege 2018, S. 129-30; Paganini 2020, S. 20-21).

Wie die oben vorgenommene Klassifizierung andeutet, gibt es aber weiterhin zu wenig Beiträge, in denen dieser Anspruch konsequent umgesetzt würde. Nur in den seltensten Fällen gelangen die Analysen also zu dem, worum es der Medienethik als einer angewandten Ethik doch eigentlich gehen sollte. Gerade angesichts der Tendenz zur öffentlichen Medienkritik und der Tatsache, dass viele Schlagworte - wie fake news, alternative Fakten, ,Lügen-Presse“ oder auch political correctness - durch implizite oder explizite moralische Bezüge gekennzeichnet sind und nach wissenschaftlich-systematischer Reflexion und Einordnung verlangen, ist diese Situation unbefriedigend.

In diesem Aufsatz möchte ich der Frage nachgehen, auf welche Faktoren sich der hier kritisierte Zustand der deutschsprachigen Medienethik zurückführen lässt und welche Hindernisse einer fruchtbaren multidisziplinären Auseinandersetzung mit medienethischen Fragestellungen im Wege stehen. Dass ich die englischsprachige Literatur dabei weitgehend unberücksichtigt lasse, hat seinen Grund vor allem darin, dass eine angemessene Betrachtung der relevanten Gemeinsamkeiten und Unterschiede hier nicht sinnvoll zu leisten ist. Obwohl die früh etablierte angelsächsische Medienethik der deutschen manchmal als leuchtendes Beispiel vorgehalten wird, finden sich die oben beschriebenen Tendenzen zum Teil auch in der englischsprachigen Literatur (vgl. auch Funiok 2011, S. 24). Da das Verhältnis zwischen der deutsch- und der englischsprachigen Medienethik also insgesamt komplex ist und eine eingehendere Analyse erfordern würde, erscheint die Beschränkung auf den Stand der deutschsprachigen Medienethik - die in der aktuellen Literatur nicht ohne Vorläufer ist (vgl. Funiok 2015; Krainer 2015) - sinnvoll. Insbesondere im nächsten Kapitel werde ich jedoch auf einige relevante Unterschiede zwischen der deutschsprachigen und der englischsprachigen Medienethik-Diskussion zu sprechen kommen.

Die These, die ich im Folgenden vertreten möchte, lautet, dass sich die deutschsprachige Medienethik mit einer Reihe fragwürdiger theoretischer Vorannahmen und Festlegungen selbst das Leben schwer macht. Die betreffenden Vorannahmen haben den Charakter von Dogmen, die weitgehend unhinterfragt akzeptiert und unzureichend diskutiert werden. Sie bewirken aber eine beträchtliche Engführung des medienethischen Diskurses und entziehen der Formulierung konkreter Handlungsanweisungen tendenziell die Grundlage.

Bei den vier Annahmen, die ich kritisch diskutieren möchte, handelt es sich 
a. um einen vor allem innerhalb der Kommunikationswissenschaften kultivierten Skeptizismus in Bezug auf die Möglichkeit rationaler Moralbegründung;

b. um die Forderung, die Medienethik dürfe nicht auf die Formulierung von „Idealnormen“ abzielen, sondern müsse stattdessen „Praxisnormen“ formulieren, die sich bewusst an den gegenwärtigen Medienstrukturen und dem Arbeitsalltag von Medienakteur*innen orientieren;

c. um die Auffassung, der Begriff der Verantwortung bilde die Schlüsselkategorie der Medienethik und er verlange nach einer konsequentialistischen Moralkonzeption, sowie

d. um die These, modernes massenmediales Handeln sei aufgrund seiner organisatorischen Strukturen nicht mit Hilfe individualethischer, sondern nur mit Hilfe kollektivethischer Ansätze angemessen zu erfassen.

Meine Zielsetzung ist dabei nicht primär empirisch, sondern theoretisch. Mir geht es nicht vorrangig darum zu beweisen, dass die von mir analysierten Annahmen die Haupt- oder gar die alleinige Ursache für den gegenwärtigen Zustand der deutschsprachigen Medienethik darstellen. Stattdessen will ich zeigen, dass die Annahmen in sich fragwürdig und die daraus abgeleiteten Folgerungen keineswegs so alternativlos sind, wie dies von vielen Autor*innen suggeriert wird. Mit der angestrebten Analyse verbindet sich gleichwohl die Hoffnung, zu einer Beseitigung derjenigen Hindernisse beizutragen, die der produktiven multidisziplinären Diskussion medienethischer Fragestellungen und einer wahrhaft normativen Medienethik im Wege stehen.

\section{Ethischer Skeptizismus}

Ein Skeptizismus in Bezug auf die Möglichkeit einer rationalen Begründung moralischer Normen kennzeichnet vor allem die kommunikationswissenschaftliche Literatur, und zwar schon seit den Anfängen der deutschsprachigen Medienethik in den 1970er und frühen 1980er-Jahren. Seine zentrale Rolle verdankt er nicht zuletzt dem Einfluss der Luhmannschen Systemtheorie, welche die deutschsprachigen Kommunikationswissenschaften nachhaltig geprägt hat. Vergleichbare Zweifel sind freilich auch außerhalb der kommunikationswissenschaftlichen Literatur zum Ausdruck gebracht worden; auch hier ist Luhmanns Theorie aber oftmals ein wichtiger Bezugspunkt (vgl. z. B. Leschke 2001, S. 84).

Luhmann selbst macht sich die angedeutete Skepsis explizit zu eigen. Seine kritische Auseinandersetzung mit normativ-ethischen Ansätzen und den Ansprüchen rationaler Moralbegründung reicht bis in die 1960er-Jahre zurück (vgl. Luhmann 2008a). Luhmanns systemtheoretischer Ansatz unterscheidet strikt zwischen dem normativen bzw. evaluativen Code der Moral, der durch die Unterscheidung gutschlecht repräsentiert wird, und dem Code der Wissenschaft, der durch die Unterscheidung wahr-falsch repräsentiert wird. Vor diesem Hintergrund sieht Luhmann die Zielsetzung einer wissenschaftlichen Normenbegründung als grundsätzlich verfehlt an. Die Funktion der Ethik als einer „Reflexionstheorie der Moral“ kann für Luhmann nur darin bestehen, die Rolle eines Beobachters einzunehmen, und zwar 
eines Beobachters, der „,beobachtet, wie die an einem moralischen Diskurs Beteiligten einander wechselseitig beobachten“ (Luhmann 2008b, S. 346).

Wie Luhmann ausführt, geht diese Rolle mit einer grundsätzlichen Abkehr vom begründungsethischen Paradigma einher:

Das würde [...] einen Verzicht auf Begründung der Moral implizieren, denn alle Begründungsversuche erscheinen in der Perspektive dessen, der sie beobachtet, als paradox, als kontraintentional wirkend, als Operationen, die Notwendigkeit suchen und Kontingenz erzeugen. (Luhmann 2008b, S. 346)

Die Ethik kann die Moral nicht begründen, sie findet sie vor, und sie hat es dann mit der Problematik dieses Befundes zu tun. (Luhmann 2008b, S. 272)

Ein ähnliche Position findet sich bei kommunikationswissenschaftlichen Medienethikern wie Manfred Rühl und Ulrich Saxer, deren Veröffentlichungen der deutschsprachigen Medienethik lange die Richtung vorgegeben haben. Rühl und Saxer gehen zunächst vom Phänomen des Wertepluralismus aus. Ihre relativistische Folgerung, dass es ,die eine richtige Ethik“ oder „die eine richtige Moral“ nicht geben kann, gilt aber offensichtlich nur dann, wenn man den bei Luhmann angedeuteten Begründungspessimismus teilt. Für die wissenschaftliche Analyse der Massenkommunikation heißt das laut Rühl und Saxer, dass Medienethik nur als reine Funktionsanalyse möglich ist:

Pluralisierung der Gesellschaft heißt vor allem, daß es die Ethik und die Moral nicht geben kann.

Da keine fertigen Ethiktheorien aus Moraltheologie und Moralphilosophie in die Kommunikationswissenschaft zu importieren sind, stellt sich dieser Wissenschaft das Problem, einen Ausgangspunkt für die Analyse von journalistischer und Medienethik im Rahmen pluraler Gesellschaften zu nennen.

Wenn also keine „bestehende“ Ethiktheorie als gegebene Struktur brauchbar ist, dann ist zu fragen nach der Funktionalität von Ethik für Journalismus und Massenkommunikation [...]. (Rühl und Saxer 1981, S. 475-77)

Obwohl der Systemtheorie in der deutschsprachigen Kommunikationswissenschaft heute nicht mehr die dominierende Rolle zukommen mag, die sie in der Vergangenheit gespielt hat, ist die durch Luhmann bewirkte Prägung im Theorie- und Methodenverständnis doch zweifelsohne noch spürbar. Sie wirkt entsprechend weiterhin in die Medienethik hinein - und sei es nur, dass genuin normative Perspektiven von kommunikationswissenschaftlichen Medienethiker*innen gar nicht ernsthaft erwogen werden. Selbst in der neuesten Literatur finden sich zudem noch Beispiele für eine Annäherung an medienethische Fragestellungen auf systemtheoretischer Grundlage (vgl. Bechthold-Hengelhaupt 2021). Entsprechend grenzen sich Autor*innen, die die Notwendigkeit einer stärker normativen Ausrichtung der kommunikationswissenschaftlichen Medienethik fordern, gezielt vom Luhmannschen Erbe ab (vgl. etwa Zurstiege 2018, 130-31).

$\mathrm{Zu}$ betonen ist darüber hinaus, dass der skizzierte Begründungspessimismus und der daraus resultierende deskriptiv-funktionalistische Zugang zu medienethischen Fragestellungen in den Kommunikationswissenschaften auch auf Grundlage anderer einflussreicher Meta-Theorien wie etwa des Radikalen Konstruktivismus vertreten 
worden sind - und weiterhin vertreten werden (vgl. etwa Weischenberg 2018). Wie Rühl und Saxers Bezugnahme auf den Pluralismus zeigt, kann die betreffende Sicht auf Moral und Moralbegründung zudem auch außerhalb solcher spezifischer Ansätze Plausibilität beanspruchen, sofern man nämlich die unleugbare Tatsache des Wertepluralismus im Sinne eines ethischen Relativismus ausdeutet, wie er unter Sozialwissenschaftler*innen traditionell beträchtliche Popularität genießt.

Die von Luhmann, Rühl und Saxer zum Ausdruck gebrachte Begründungsskepsis hat nun ohne Zweifel einen wahren Kern. Dieser Kern besteht in der Unmöglichkeit einer Letztbegründung der Moral. Angesichts des prinzipiellen Unterschiedes von Seinsaussagen und Sollensaussagen lassen sich moralische Normen und Prinzipien nicht aus Fakten, d.h. aus rein deskriptiven Annahmen, ableiten. Ebenso wenig lassen sie sich, allen Versuchen zum Trotz, auf evidente erste normative Prinzipien zurückführen. Die Idee, moralische Urteile ließen sich deduktiv-nomologisch aus unbestreitbaren ersten Prämissen ableiten, erscheint daher in der Tat verfehlt.

Daraus folgt aber mitnichten, dass jede Form rationaler und intersubjektiv nachvollziehbarer Moralbegründung zum Scheitern verurteilt wäre. Die Unmöglichkeit einer Letztbegründung der Moral ist in der wissenschaftlichen Ethik weithin anerkannt. Aus eben diesem Grund sind fundamentalistische Begründungsansätze schon seit den frühen 1970er-Jahren mehr und mehr durch kohärentistische Modelle ersetzt worden sind, und zwar nicht nur in der Moralphilosophie und der politischen Philosophie, sondern auch und gerade in multidisziplinär geprägten Debatten der angewandten Ethik. Diese Tatsache wird nicht nur von Luhmann, Rühl und Saxer ignoriert. Die Existenz und das Potenzial solcher Ansätze sind ganz allgemein in der kommunikationswissenschaftlichen Literatur und der Medienethik lange Zeit nicht hinreichend gewürdigt worden.

Kohärentistische Begründungsmodelle stellen eine wichtige Alternative zu deduktivistischen Modellen (oder top-down approaches) auf der einen und kontextualistischen Modellen (oder bottom-up approaches) auf der anderen Seite dar (vgl. hierzu auch Bayertz 2008; Schöne-Seifert 2011). Während bei ersteren ein moralisches Prinzip oder Urteil aus einem hierarchisch übergeordneten allgemeineren Prinzip logisch abgeleitet wird, gehen letztere von Einzelfallbewertungen aus und versuchen, neue Fälle in Analogie zu bereits beurteilten Fällen zu entscheiden. Das kohärentistische Modell versucht demgegenüber, Prinzipien mit unterschiedlicher Reichweite und verschiedene Einzelfallbewertungen in einen kohärenten Gesamtzusammenhang zu bringen und dadurch ein „Überlegungsgleichgewicht“ herzustellen (vgl. Rawls 1971). Sollte es im Sinne der Kohärenz erforderlich sein, können dabei sowohl allgemeinere Prinzipien als auch Einzelfallbewertungen angepasst oder aufgegeben werden.

$\mathrm{Zu}$ den Bereichen der angewandten Ethik, in denen kohärentistische Begründungsmodelle Verwendung finden, zählt seit der Veröffentlichung des Buches Principles of Biomedical Ethics von Tom Beauchamp und James Childress (2019) vor allem die Bio- und Medizinethik. Eine Grundidee des darin entwickelten prinzipilistischen Ansatzes besteht darin, von vier Prinzipien mittlerer Reichweite auszugehen, nämlich dem Autonomieprinzip (respect for autonomy), dem Nichtschadensprinzip (nonmaleficence), dem Fürsorgeprinzip (beneficence) und dem Gerechtigkeitsprinzip (justice). Anstatt also zu fragen, wie konkrete moralische Probleme auf der 
Grundlage eines einzigen und fundamentalen ethischen Prinzips wie des kategorischen Imperativs oder des Nützlichkeitsprinzips zu lösen sind, greifen Beauchamp und Childress auf mehrere, grundsätzlich gleichberechtigte Prinzipien und Werte zurück, die in verschiedenen Ethikkonzeptionen ihren Platz haben und daher von Vertretern unterschiedlicher umfassender Lehren gleichermaßen akzeptiert werden können.

Auf Grundlage der genannten Prinzipien werden normative Handlungsregeln für die biomedizinische Praxis abgeleitet, die in Form konkreterer Handlungsanweisungen weiter ausbuchstabiert werden. Im Fall des Autonomieprinzips ist dies unter anderem die Regel, dass Patient*innen nur dann einer Behandlung unterzogen werden dürfen, wenn ihre informierte Einwilligung (informed consent) vorliegt. Zu den Regeln, die sich aus dem Nichtschadensprinzip und dem Fürsorgeprinzip als seinem positiven Gegenstück ergeben, gehören das Verbot, Patient*innen Schmerz oder Leiden zuzufügen, und das Gebot, Menschen in Not zu retten. Im Kontext des Gerechtigkeitsprinzips schließlich ist die Regel angesiedelt, medizinische Ressourcen fair zu verteilen.

Die Eigenheiten des kohärentistischen Begründungsmodells zeigen sich dabei besonders an der Art und Weise, in der Konflikte zwischen den vier Prinzipien bzw. den daraus abgeleiteten Handlungsregeln aufgelöst werden. Wie Beauchamp und Childress ausdrücklich betonen, sind die Prinzipien nicht hierarchisch geordnet. Alle Prinzipien sind grundsätzlich in der Lage, die jeweils anderen zu übertrumpfen. Was mit Blick auf einen konkreten Fall, wie etwa die Sterbehilfe, das Resultat der Abwägung ist, entscheidet sich danach, welche der alternativen Handlungsweisen insgesamt am besten mit den relevanten Prinzipien, Regeln und wohlerwogenen Einzelurteilen in einen kohärenten Gesamtzusammenhang gebracht werden kann.

Auch ohne den prinzipilistischen Ansatz als Allheilmittel für die Probleme rationaler Moralbegründung präsentieren oder die Fortschritte in der Bio- und Medizinethik der letzten Jahrzehnte überschätzen zu wollen, kann kaum geleugnet werden, dass die von Beauchamp und Childress eingebrachte Perspektive der Debatte insgesamt sehr gut getan hat. Es ist nicht zu sehen, warum die Medienethik nicht ähnlich verfahren sollte. Auch in der Medienethik geht es nicht darum, die Moral von Grund auf neu zu erfinden oder zuallererst die Notwendigkeit der Moral zu beweisen. Wie die in der Einleitung beschriebenen Beispiele deutlich machen, geht es eher darum, Prinzipien und Werte, die von den Teilnehmern des Diskurses typischerweise akzeptiert werden, wie Respekt und Unparteilichkeit, Autonomie und Solidarität oder auch Meinungs- und Pressefreiheit, allgemeines Persönlichkeitsrecht und Recht auf Privatleben, in einen kohärenten Zusammenhang zu bringen und festzustellen, welches Verhalten sie mit Blick auf konkrete Entscheidungssituationen fordern und wie mögliche Konflikte plausibel aufgelöst werden können.

Dass bislang kaum Versuche unternommen worden sind, den prinzipilistischen Ansatz und das damit verbundene kohärentistische Begründungsverfahren für medienethische Fragestellungen fruchtbar zu machen, muss daher überraschen. Umso erstaunlicher erscheint es angesichts der Tatsache, dass Beauchamp mit dem zusammen mit Stephen Klaidmann verfassten Buch The Virtuous Journalist selbst eine Monographie zu einem medienethischem Thema veröffentlicht hat, in dem der prinzipilistische Ansatz allerdings nicht mit der gleichen systematischen Ausführ- 
lichkeit erläutert und entwickelt wird wie in Principles of Biomedical Ethics (vgl. Klaidmann und Beauchamp 1987). Erst in letzter Zeit ist dem Beitrag, den prinzipilistische Ansätze (oder hermeneutisch-rekonstruktive Ansätze ganz allgemein) zu leisten vermögen, mehr Aufmerksamkeit geschenkt und mit der Entwicklung entsprechender medienethischer Konzeptionen begonnen worden.

Dieser gerade erst begonnenen Erschließung prinzipilistischer Ansätze für die Medienethik ist eine Phase vorausgegangen, in der die Probleme fundamentaltheoretischer Ansätze zwar erkannt, in der aus dieser Erkenntnis aber nicht die richtigen Schlüsse gezogen wurden. Die Gültigkeit solcher Fundamentaltheorien, zu denen neben der aristotelischen Tugendethik, der Kantischen Pflichtenethik und dem Utilitarismus auch die Diskursethik und der an Thomas Hobbes angelehnte ethische Kontraktualismus gezählt werden können, ist notorisch umstritten, weswegen medienethische Konzeptionen, die auf einem dieser Ansätze aufbauen, nur bedingt anschlussfähig sind.

In Reaktion auf dieses Problem ist, etwa von Rüdiger Funiok und Bernhard Debatin, die Entwicklung einer ,systematischen Kombination“ (Debatin und Funiok 2003, S. 13) verschiedener normativer Theorien angeregt worden (vgl. auch Karmasin und Litschka 2014, S. 374). Dabei wurde aber nicht ausreichend anerkannt, dass eine Kombination etwa der Kantischen Moralphilosophie und des Utilitarismus streng genommen unmöglich ist, weil es sich bei beiden Theorien um monistische Theorien handelt, die den Anspruch erheben, mit dem kategorischen Imperativ bzw. dem Nützlichkeitsprinzip das eine gültige und fundamentale Prinzip der Moral formuliert zu haben. Eine Unvereinbarkeit besteht auch zwischen deontologischen und konsequenzialistischen Theorien ganz allgemein, wie sie von Debatin und Funiok explizit ins Spiel gebracht wird, da die Grundthesen beider Ansätze einander strikt entgegengesetzt sind (siehe hierzu auch Abschn. 4).

Veröffentlichungen zur Medienethik, die für sich reklamieren, die genannten Theorien oder andere historisch einflussreiche Ansätze der allgemeinen normativen Ethik miteinander zu verbinden, liefern entsprechend in den meisten Fällen überhaupt keine systematisch entwickelte normative Theorie, sondern behandeln die Ansätze eher wie einen Ideenfundus, aus dem man sich je nach Bedarf bedienen kann. Um eine Verträglichkeit der verschiedenen Ansätze erreichen zu können, muss man, wie Beauchamp und Childress es tun, die Ebene fundamentaler Prinzipien verlassen und sich auf Prinzipien mit geringerer Reichweite konzentrieren, die grundsätzlich nebeneinander Bestand haben können.

Stimmen, die dieses Vorgehen für vielversprechend halten, finden sich vor allem in der englischsprachigen Literatur. Ein wichtiger Unterschied gegenüber der deutschsprachigen Medienethik-Diskussion besteht darin, dass die Herausforderung, die sich aus der Tatsache des Wertepluralismus ergibt, hier eingehender thematisiert und theoretisch zu überwinden versucht wird (vgl. etwa Christians 2013; Hanitzsch et al. 2013; Metz 2018; Nikolaev 2018; Christians 2019; Cooper und Christians 2020). Als Folge hieraus sind die verschiedenen methodischen Optionen ethischer Rechtfertigung (top-down, bottom-up, Holismus) in der englischen Medienethik früher und ausführlicher diskutiert worden (vgl. beispielsweise Ward 2011; Couldry 2013; Metz 2018). Auch hier gibt es freilich bislang nur wenige ausgearbeitete Konzeptionen, die den prinzipilistischen Ansatz konsequent umsetzen. 
Eines der wenigen Beispiele liefert die kürzlich von Fabian Medvecky und Joan Leach (2019) vorgelegte Monographie zur Ethik der Wissenschaftskommunikation. Ihr Entwurf beruht auf vier Prinzipien mittlerer Reichweite (utility, accuracy, kairos, generosity), die mit Blick auf relevante Handlungssituationen konkretisiert werden. Im deutschen Sprachraum liegt mit der Monographie Werte für die Medien(ethik) von Claudia Paganini (2020) ebenfalls ein erster ausführlicherer prinzipilistischer Beitrag zur Kommunikations- und Medienethik vor (vgl. auch bereits Paganini 2018). Im Mittelpunkt steht dabei ein „Kernbestand“ (Paganini 2020, S. 90) an Werten, zu dem Transparenz, Fairness, Respekt, Verantwortung und Kompetenz zählen.

Wie Medvecky und Leach selbst betonen, können ihre Überlegungen nur einen ersten Schritt auf dem Weg zu einer prinzipilistischen Ethik der Wissenschaftskommunikation leisten (vgl. Medvecky und Leach 2019, S. 4 und 13). Hinzu kommt jedoch, dass ihr Beitrag in inhaltlicher und methodischer Hinsicht unbefriedigend bleibt, und Ähnliches gilt für den Ansatz von Paganini. Das Hauptproblem besteht darin, dass die ausgewählten Prinzipien kaum in Form spezifischerer Handlungsregeln und Handlungsanweisungen konkretisiert werden: Mögliche normative Konsequenzen für spezifische Arbeitsfelder werden zwar hie und da exemplarisch angedeutet, aber nicht konsequent entwickelt.

Im Falle von Medvecky und Leach liegt dies vor allem an ihrem spezifischen Verständnis des Prinzipilismus. Ihrer Auffassung zufolge geht es dem prinzipilistischen Ansatz letztlich nicht um Antworten auf die Frage „Wie soll ich handeln?“, sondern darum, allgemeinere Prinzipien zu formulieren, mit deren Hilfe sich ethische Probleme „durchdenken“ lassen. Ironischerweise berauben Medvecky und Leach den eigentlich fruchtbaren Ansatz von Beauchamp und Childress damit seines wahren Potenzials.

Im Fall Paganinis trägt nicht zuletzt die Auswahl der Werte zum Mangel an normativer Konkretisierung bei, die im Übrigen - wie bei Medvecky und Leach - auch ansonsten nicht vollends zu überzeugen vermag. So findet sich mit der Verantwortung ein Konzept in Paganinis Kernbestand, bei dem es sich gar nicht um einen substanziellen Wert handelt, sondern um ein sogenanntes thin moral concept, aus dem sich ohne die Ergänzung materialer Wertbestimmungen keine Handlungsanleitungen gewinnen lassen. Eine Ergänzung erfordern auch die zunächst unbestimmten Begriffe des Respekts und der Kompetenz.

Die von Medvecky und Leach vorgeschlagenen Werte weisen demgegenüber einen engeren Handlungsbezug auf. Aufgrund des schon skizzierten methodischen Grundansatzes wird dieser Bezug aber nicht wirklich genutzt. Problematisch erscheint darüber hinaus, dass sie die Bedeutung des Prinzips kairos, das dem Timing kommunikativer Aktivitäten entscheidende Bedeutung zuweist (saying the right thing at the right time), insgesamt überbetonen und umgekehrt, wie auch Paganini, dem Autonomieprinzip nur wenig Beachtung schenken. Der Begriff der Selbstbestimmung mag in der Kommunikationswissenschaft bislang nicht die Rolle spielen, die ihm in Nachbardisziplinen zukommt. Er stellt aber ohne Zweifel einen zentralen Begriff der angewandten Ethik und der normativen Ethik ganz allgemein dar. Es ist zudem vermehrt darauf hingewiesen worden, dass Vorstellungen von Autonomie, Selbstbestimmung und Souveränität häufig als impliziter normati- 
ver Bezugspunkt auch der kommunikationswissenschaftlichen Forschung fungieren (vgl. Wessler et al. 2018, S. 395). ${ }^{3}$

Die Nichtberücksichtigung des Autonomiebegriffs erscheint daher fragwürdig, und besonders gilt dies für Medvecky und Leachs These, der Begriff besitze mit Blick auf die ethischen Aspekte der Wissenschaftskommunikation keine besondere Relevanz (vgl. Medvecky und Leach 2019, S. 88). Die Relevanz derjenigen Prinzipien, die stattdessen in den Mittelpunkt gerückt werden, wird zudem nicht eigens begründet. Der von Paganini gewählte Zugang, den Kernbestand an Werten über eine Analyse von Selbstverpflichtungskodizes zu identifizieren, erscheint hier angemessener. Die Idee der hermeneutischen oder rekonstruktiven Moralbegründung derartig eng auszulegen, ist aber beileibe nicht zwingend und birgt Gefahren, auf die ich im folgenden Abschnitt noch einmal zu sprechen kommen werde.

Eine Alternative bestünde darin, sich dem spezifischen Handlungskontext Medien zunächst allgemeiner zu nähern, wie dies Beauchamp und Childress auch mit Blick auf den Handlungskontext Medizin tun, und einen Kanon substanziellerer moralischer Werte zu identifizieren, die für das normative Selbstverständnis moderner demokratischer Gesellschaften kennzeichnend sind und ihren Niederschlag in verfassungsrechtlichen Regelungen gefunden haben. Erst in einem zweiten Schritt könnten dann Bezüge zu bereits existierenden medienethischen Kodifizierungen hergestellt werden, wobei Letztere zugleich im Lichte des allgemeineren Wertekanons kritisch reflektiert werden könnten.

Obwohl also umfangreichere Arbeiten vorliegen, in denen die prinzipilistische Herangehensweise für die Kommunikations- und Medienethik fruchtbar gemacht werden soll, ist das Potenzial, das dem Ansatz mit Blick auf die rationale Begründung ethischer Bewertungen und die Formulierung konkreter Handlungsanweisungen zukommt, noch weitgehend ungenutzt. Auch die Frage, welche Prinzipien mittlerer Reichweite dem Themenfeld öffentliche Kommunikation am angemessensten sind, bleibt noch unbeantwortet. Unklar ist dabei insbesondere, warum die von Beauchamp und Childress verwendeten Prinzipien nicht auch für kommunikationsund medienethische Zusammenhänge einen sinnvollen Startpunkt liefern sollten. Bei aller Kritik machen die vorliegenden Ansätze zu einer prinzipilistischen Medienethik jedoch einmal mehr deutlich, dass die Auffassung, eine wissenschaftliche Medienethik müsse jeglichem Anspruch auf Moralbegründung abschwören, nicht nur voreilig, sondern schlicht falsch ist.

\section{Ideal- vs. Praxisnormen}

Der zweite ,Irrtum“, den ich ansprechen möchte, hängt mit der begrifflichen Unterscheidung von „Idealnormen“ und „Praxisnormen“ zusammen. Diese Unterscheidung, die vor allem auf Dieter Birnbacher zurückgeht, wird in zahlreichen aktuelle-

\footnotetext{
3 Zur Bedeutung des Autonomiebegriffs für eine Medienethik in Zeiten der Digitalisierung siehe auch bereits Koska (2015, S. 85). Zu Versuchen, den Begriff für spezifische Kontexte medialen Handelns fruchtbar zu machen, siehe die Beiträge in dem von Wessler, Haffer und Rinke herausgegebenen Themenheft „Selbstbestimmung in der digitalen Welt".
} 
ren Beiträgen zur Medienethik aufgegriffen, wobei unisono die Auffassung vertreten wird, das Ziel der Medienethik könne nicht in der Formulierung von Idealnormen bestehen, sondern nur in der Formulierung von Praxisnormen. Eine entsprechende These findet sich etwa in den medienethischen Veröffentlichungen von Christian Schicha und Carsten Brosda:

Ideale Normen sind [...] das Resultat abstrakter normativ-ethischer Überlegungen, die jedoch auf dieser idealen Ebene keine praktische Hilfe bei konkreten Handlungsentscheidungen liefern können. Sie sind zu allgemein, zu unbestimmt und zu rigide, um faktisch als Regeln für die konkrete Lebenspraxis dienen zu können. (Schicha 2010b, S. 29)

Idealnormen müssen deshalb in Praxisnormen übersetzt werden, also in bereichsspezifische „Durchführungsregeln“, die den historischen und sozioökonomischen Rahmen berücksichtigen. Sie tragen legitimer Weise einer Anpassung an gesellschaftliche oder individuelle Einschränkungen der Normbefolgungsmöglichkeiten Rechnung, ohne sich jedoch zu stark an opportunistischen Gepflogenheiten in der Praxis zu orientieren. (Brosda und Schicha 2010, S. 11-12)

Die hier eingeführte begriffliche Unterscheidung mag zunächst harmlos erscheinen, und Ähnliches gilt für die daraus abgeleitete Forderung nach praxisbezogener Konkretisierung. Es ist aber wichtig zu betonen, dass diejenigen Autor*innen, die die Unterscheidung mit Blick auf die Medienethik stark machen, typischerweise über die eben zitierten, eher moderaten Forderungen hinausgehen. Brosda und Schicha liefern hier selbst das beste Beispiel:

Nicht wenige Akzeptanzprobleme hat sich die Medienethik aufgrund eigener Engführungen selbst zuzuschreiben: Wer nur fragt, was nicht geht, der geht anderen schnell auf die Nerven. Wer moralinsauer Verfehlungen beschreibt, bringt es kaum zum gefragten Ratgeber. Und wer im Gestus von „ex cathedra"-Sprüchen Sollensvorschriften dekretiert, die mit der Praxis kaum etwas zu tun haben, der darf sich nicht wundern, wenn er nicht ernst genommen wird. (Brosda und Schicha 2010, S. 10)

Oft sind anspruchsvolle ethische Prinzipien zu rigoros, um eine Durchsetzungschance in der Praxis zu haben. Darüber hinaus weichen sie oftmals zu gravierend von den gängigen Gegebenheiten und Konventionen der Lebenspraxis $\mathrm{ab}$, um die Akteure zur Beachtung entsprechender Prinzipien zu motivieren. (Schicha 2010b, S. 29)

Die zuerst zitierten Passagen vermitteln den Eindruck, das Problem von Idealnormen bestehe in ihrer Abstraktheit, also in dem, was jede angewandte Ethik vernünftigerweise überwinden möchte. Die zuletzt zitierten Passagen erwecken dagegen eher den Eindruck, Idealnormen seien in einem umgangssprachlichen Sinne zu „,idealistisch“, weil sie den Menschen Dinge abverlangen, die diese nicht zu leisten gewillt sind. Sie aus diesem Grund und in diesem Sinne durch leichter erfüllbare Praxisnormen ersetzen zu wollen, hieße dann aber gegebenenfalls, genau die Art von Opportunismus zu betreiben, die Brosda und Schicha offiziell ablehnen. 
Der Eindruck, dass bei der Erörterung von Ideal- und Praxisnormen ganz unterschiedliche Punkte vermischt werden, wie etwa die Allgemeinheit von Normen, ihre Unbestimmtheit, ihre Strenge und die Wahrscheinlichkeit ihrer tatsächlichen Befolgung, entsteht auch bei Stapf und Paganini (vgl. Stapf 2006, S. 27-28; Paganini 2020, S. 70-71). Bei Paganini finden sich darüber hinaus Wendungen, die an die zuvor kritisierten Aussagen Brosdas und Schichas erinnern. Dazu zählt beispielsweise die Forderung, die Medienethik dürfe nicht vorrangig belehren, die unmittelbar die Frage aufwirft, wie eine nicht belehrende Medienethik ihrer Kritik- und Orientierungsfunktion gerecht werden soll. Hier stellt sich fast unweigerlich der Verdacht ein, dass eine Art maßgeschneiderter Medienethik für die Akteur*innen der Massenmedien produziert werden soll, bei der im Großen und Ganzen alles so bleiben kann, wie es ist.

Dass das Bemühen um die Formulierung von Praxisnormen schnell in Konflikt mit der normativ-kritischen Grundausrichtung der Medienethik geraten kann, zeigt sich auch an Paganinis methodischem Vorgehen. Prinzipilistische Ansätze scheinen grundsätzlich gut geeignet, das Problem der Abstraktheit idealer Normen zu überwinden: Startet man mit substanziellen Prinzipien mittlerer Reichweite wie dem Autonomie- oder dem Nichtschadensprinzip, und eben nicht mit fundamentalen Grundsätzen wie dem kategorischen Imperativ, und versucht man darüber hinaus, die Prinzipien mit Hilfe eines kohärentistischen Verfahrens zu spezifischeren Handlungsregeln und Einzelfallbewertungen in Bezíehung zu setzen, dann ergibt sich der geforderte Praxisbezug fast automatisch. Gerade weil es im prinzipilistischen Ansatz immer auch um die Frage geht, wie sich mögliche Kollisionen von Prinzipien, Regeln und Einzelfallbewertungen in kohärenter Weise auflösen lassen, sind die ethischen Bewertungen, die im Zuge eines solchen Ansatzes gewonnen werden, notwendig auf spezifische Handlungszusammenhänge zugeschnitten.

Wenn man dagegen eine weitergehende Anpassung an die zu reflektierende Praxis vornimmt und bestimmte Handlungsstrukturen oder Überzeugungen medialer Akteur*innen implizit oder explizit für sakrosankt erklärt, gibt man den wissenschaftlichen Anspruch des kohärentistischen Modells auf und entfernt sich vom Verständnis rationaler Moralbegründung, das ihm seit jeher zugrunde liegt: Schon Rawls betont, dass selbst unsere fundamentalen moralischen Intuitionen bei dem Bemühen um ein Überlegungsgleichgewicht immer wieder neu geprüft und notfalls aufgegeben werden müssen (vgl. Rawls 1971, S. 20). Erst recht muss dies für die strukturellen Rahmenbedingungen medialen Handelns gelten, die oftmals keine genuin moralische, sondern eine wirtschaftliche Grundlage haben.

Obwohl nun Paganini eine bloß affirmative Medienethik ausdrücklich zurückweist (vgl. Paganini 2020, S. 79), kann sie aufgrund ihrer spezifischen Umsetzung der hermeneutisch-rekonstruktiven Methode eine weitreichende Affirmation der geltenden Praxis doch kaum vermeiden. Paganini identifiziert ihren Kernbestand an Werten anhand von Selbstverpflichtungskodizes, die aus verschiedenen Kontexten medialen Handelns stammen. Die Möglichkeit, vom außermedialen Normkontext der Gesellschaft auszugehen, wird auf diese Weise verschenkt. Einer radikaleren Medienkritik, wie sie für eine wissenschaftliche Medienethik immer eine Option sein sollte und nach Auffassung mancher Autor*innen gerade in Zeiten großer gesellschaftlicher Umwälzungen wie der Digitalisierung gefordert ist (vgl. Ward 2015), wird damit 
von vornherein die Grundlage entzogen. In letzter Konsequenz wird die Medienethik so in einen deskriptiv-ethischen Ansatz hineingedrängt, dem es lediglich um die systematische Rekonstruktion der gegenwärtigen Medienmoral zu tun ist. Das aber ist etwas völlig anderes als das, was die Medienethik als angewandte Ethik leisten soll und leisten kann.

Wenn sie so wie bei Brosda und Schicha oder Paganini aufgefasst und methodisch umgesetzt wird, trägt die Unterscheidung von Ideal- und Praxisnormen dazu bei, die Medienethik in eine falsche Richtung zu lenken. Obwohl sie einen wahren Kern hat, stellt sie in Form der hier diskutierten Varianten eines der Hindernisse da, das es im Sinne einer genuin normativen Medienethik zu überwinden gilt.

\section{Verantwortung und Konsequenzialismus}

Ein dritter „Irrtum“, der in der gegenwärtigen medienethischen Diskussion eine Rolle spielt, besteht in der Sichtweise, der Begriff der Verantwortung sei für die Medienethik (oder gar für jede Form angewandter Ethik) fundamental, und eine angemessene medienethische Konzeption müsse daher konsequenzialistisch angelegt sein. Der Irrtum liegt hier weniger in der Ansicht, der Verantwortung komme entscheidende Bedeutung zu, als in dem, was mit Blick auf die ethische Theoriebildung daraus abgeleitet wird. Gleichwohl lässt sich mit guten Gründen die Auffassung vertreten, dass Relevanz und Potenzial des Verantwortungsbegriffs in der aktuellen medienethischen Debatte insgesamt überschätzt werden.

Die Verbindung der beiden genannten Annahmen findet sich explizit bei Mathias Rath. Rath charakterisiert die Medienethik unter Bezugnahme auf Webers berühmte Unterscheidung von Gesinnungs- und Verantwortungsethik zunächst als Verantwortungsethik:

Medienethik ist Verantwortungsethik, und zwar in einem sehr viel radikaleren Sinne, als ihn Max Weber [...] bei der Einführung dieses Terminus gedacht hat. „Verantwortung“ als ethische Kategorie meint einen umfassenderen Anspruch [...]. Verantwortung heißt nicht nur ethische Orientierung an den Folgen, sondern ethische Verpflichtung, das ganze Feld möglicher Folgen zu erfassen. (Rath 2010, S. 143)

Daraus leitet er ab, dass ein angemessener Umgang mit diesem Aspekt der Folgenverantwortung einen konsequenzialistischen Ansatz erfordert:

Medienethik ist konsequentialistische Ethik. Das heißt, für applied ethics ist die Frage wichtig, welche Folgen Handlungen zeitigen. Ethiken, die diese Frage [...] prinzipiell ausschließen, beziehen sich allein auf Gesinnung oder Pflichten des Handelnden. Die lebensweltlichen Probleme können von diesen ,deontologisch" genannten Ethiken zwar konstatiert, aber nicht bearbeitet werden. (Rath 2010, S. 143)

Obwohl die Beschränkung auf konsequenzialistische Ansätze nur selten mit dieser Deutlichkeit postuliert wird, finden sich doch bei zahlreichen Autor*innen ähnliche Überlegungen. Die Behauptung, der Begriff der Verantwortung sei für die 
Medienethik zentral, stellt innerhalb der einschlägigen Literatur geradezu einen Gemeinplatz dar (vgl. etwa Funiok 2002, S. 44; Krainer 2002, S. 156; Debatin 2004, S. 39; Stapf 2006, S. 10; Alsdorf 2007, S. 12 und 29; Friedrichsen und Gertler 2011, S. 86; Thies 2011, S. 207; Altmeppen et al. 2013, S. 281; Karmasin und Litschka 2014, S. 375; Weischenberg 2018, S. 1). Dass eine besondere Nähe zwischen dem Verantwortungsbegriff und dem Konsequenzialismus besteht, wird sowohl innerhalb als auch außerhalb des medienethischen Diskurses immer wieder suggeriert, oftmals - wie bei Rath - mit Verweis auf Webers Unterscheidung des gesinnungsethischen und des verantwortungsethischen Paradigmas (vgl. Bayertz 1995, S. 47; Birnbacher 1995, S. 147-48; Teichert 1996, S. 821-22; Funiok 2002, S. 43; Alsdorf 2007, S. 39; Friedrichsen und Gertler 2011, S. 70; Hinsch 2017, S. 106).

Die auf diese Weise nahegelegte Beschränkung der medienethischen Theoriebildung wäre in zweierlei Hinsicht bedenklich. Zum einen würde die medienethische Debatte inhaltlich in erheblicher Weise limitiert, mit der Folge, dass das Potenzial nicht-konsequenzialistischer Positionen nicht ausgelotet geschweige denn ausgeschöpft würde. Zum anderen würde die Medienethik die allgemeineren Probleme „erben“, denen sich konsequenzialistische Ethiken gegenüber sehen und die von Kritikern immer wieder herausgestellt worden sind. Hier sind vor allem Probleme in der praktischen Anwendung zu nennen, die sich aus der Unmöglichkeit ergeben, alle relevanten Folgen einer Handlung oder Verhaltensweise im Vorhinein zu identifizieren und im Sinne eines umfassenderen Kalküls zueinander in Beziehung zu setzen. Erwähnenswert ist zudem, dass die Medienethik auf diese Weise in eine Richtung gerückt würde, die im deutschen Sprachraum nie wirklich populär war und in Form ihrer wichtigsten Variante, des auf Bentham und Mill zurückgehenden Utilitarismus, bis heute kritisch beäugt und als Bedrohung verfassungsrechtlich garantierter Grundfreiheiten wahrgenommen wird.

Trotz dieser Bedenken könnte eine Fokussierung oder gar Beschränkung auf das konsequenzialistische Paradigma freilich der Sache nach richtig und gerechtfertigt sein. Im Folgenden möchte ich zeigen, warum sie dies nicht ist. Wie die zuletzt zitierte Passage andeutet, basiert Raths Auffassung auf der Einschätzung, dass sich Verantwortungsbegriff und Pflichtbegriff wesentlich unterscheiden. Es ist Rath zuzugestehen, dass die jüngere Literatur zum Verantwortungsbegriff eine derartige Trennung durchaus nahelegt. So hat beispielsweise Kurt Bayertz in verschiedenen Veröffentlichungen die Auffassung vertreten, der Verantwortungsbegriff erfülle eine Funktion, die der Pflichtbegriff nicht (mehr) in dieser Form erfüllen könne, und genau hier liege der Grund, warum der Verantwortungsbegriff seit dem späten 19. Jahrhundert zunehmend an Bedeutung gewonnen habe.

Laut Bayertz haben Industrialisierung, Technisierung und Arbeitsteilung dazu geführt, dass viele moralisch relevante Ereignisse nicht mehr eindeutig einem individuellen Handelnden zugerechnet und als Ergebnis einer individuellen Pflichtverletzung begriffen werden können (vgl. Bayertz und Beck 2017, S. 139). In dieser veränderten historischen Situation werde der Pflichtbegriff zunehmend durch den offeneren Begriff der Verantwortung ersetzt:

Pflichten sind explizite Handlungsvorschriften, mit denen mehr oder weniger präzise festgelegt wird, was zu tun ist. Mit dem Verantwortungsbegriff in seiner 
nichtklassischen Bedeutung werden demgegenüber bestimmte positiv ausgezeichnete (erwünschte) Zustände umschrieben, ohne daß im einzelnen festgelegt werden muß, wie der jeweilige Verantwortliche diese Zustände herbeiführt oder aufrechterhält. (Bayertz 1995, S. 33)

Wie immer man Bayertz' entwicklungsgeschichtliche These letztlich bewerten mag: Es ist fraglich, ob sich die Kontrastierung von Verantwortung und Pflicht, die auch von anderen Autor*innen angedeutet und mit Blick auf medienethische Fragestellungen aufgegriffen worden ist (vgl. etwa Werner 2020, S. 38-39), aufrechterhalten werden kann, und zwar aus zwei Gründen. Zum einen kann auch der Pflichtbegriff in der von Bayertz und anderen Autor*innen beschriebenen Weise auf Ereignisse oder Zustände bezogen werden. So können wir etwa Eltern die Pflicht zuschreiben, für das Wohlergehen ihrer Kinder zu sorgen, ohne dass dabei schon spezifiziert werden müsste, was genau sie in konkreten Situationen dafür zu tun haben. Legt man das von Immanuel Kant in der Metaphysik der Sitten skizzierte Verständnis zugrunde, handelt es sich hier um „unvollkommene Pflichten“, und ein zentrales Versäumnis der jüngeren Debatte um das Verhältnis von Pflicht und Verantwortung besteht darin, der Unterscheidung von vollkommenen und unvollkommenen Pflichten zu wenig Aufmerksamkeit geschenkt zu haben (vgl. allerdings Albertzart 2015).

Zum anderen ist überhaupt nicht klar, ob eine plausible Konzeption moralischer Verantwortung ohne einen robusten Begriff moralischer Pflicht auskommen kann. Die moralische Verantwortung, bestimmte Weltzustände herbeizuführen oder zu verhindern, lässt sich einer Person nur dann legitimerweise zuschreiben, wenn sie auch über die Möglichkeit verfügt, durch ihre Handlungen einen kausalen Beitrag dazu zu leisten (vgl. auch Werner 2020, S. 42). Sobald diese Möglichkeit aber gegeben ist, spricht wenig dagegen, der Person zusätzlich eine Pflicht zur Ausführung oder Unterlassung der betreffenden Handlungen zuzuschreiben. Mehr noch: Nimmt man die praktische Funktion der Medienethik als einer angewandten Ethik ernst, erscheint eine solche Konkretisierung der moralischen Verantwortung von Medienschaffenden im Sinne der Handlungsorientierung unverzichtbar.

Gerade weil man also Personen und Institutionen Verantwortung für alles Mögliche zuschreiben kann, ohne dabei schon sagen zu müssen, welche konkreten normativen Konsequenzen sich aus der Verantwortung jeweils ergeben (vgl. Altmeppen 2020, S. 87), trägt die aktuelle Popularität des Verantwortungsbegriffs dazu bei, dass es in der medienethischen Literatur so selten zur Formulierung eindeutiger Handlungsanweisungen kommt. So richtig es sein mag, dass der Medienethik mit starren, kontextunabhängigen und sehr allgemein gehaltenen Pflichtenkatalogen wenig geholfen wäre, so wenig folgt doch daraus, dass die Idee individualisierbarer Handlungspflichten in der Medienethik keine zentrale Rolle spielen sollte.

Man mag an dieser Stelle einwenden, dass, wer den Pflichtbegriff in dieser offeneren und kontextsensitiveren Weise verwendet, eben nicht mehr mit dem Pflichtbegriff deontologischer Ethiken operiert. Die Tatsache, dass der Begriff der unvollkommenen Pflicht seinen festen Platz in der Kantischen Moraltheorie hat, sollte aber bereits ernste Zweifel an diesem Einwand aufkommen lassen. Zudem ist es wichtig, auf ein weiteres grundsätzliches Problem von Raths Darstellung hinzuweisen, nämlich auf 
eine übergriffige und letztlich unangemessene Verwendung des Ausdrucks „Konsequenzialismus“, die auch viele andere medienethische Beiträge kennzeichnet.

Die Unterscheidung von Deontologie und Konsequenzialismus wird in der medienethischen Debatte sehr stark mit Webers Unterscheidung zwischen Gesinnungsund Verantwortungsethik begründet bzw. schlicht mit dieser gleichgesetzt. Eine derartige Annäherung ist aber wenig hilfreich. Die Unterscheidung zwischen deontologischen und konsequenzialistischen Ethiken haben Autoren wie C. D. Broad und William Frankena etabliert. Nach ihrer Ansicht sind Moraltheorien konsequenzialistisch, wenn sie die moralische Bewertung von Handlungen ausschließlich von den Handlungsfolgen abhängig machen. Alle anderen Theorien, also auch solche, die den Folgen große, aber eben nicht alleinige Bedeutung zuschreiben, sind deontologisch.

Was Weber als Gesinnungsethik beschreibt, nämlich eine moralische Haltung, die gar nicht auf Folgen, sondern nur auf die Gesinnung des Handelnden schaut, stellt folglich nur eine mögliche Variante des deontologischen Ansatzes dar (und zwar eine sehr extreme und sehr unplausible). Viele andere deontologische Theorien weisen den Handlungsfolgen sehr wohl eine wichtige Rolle zu, ohne deshalb konsequenzialistisch zu sein. Es ist nicht einzusehen, warum solche Ansätze für die Medienethik keine wichtigen Beiträge liefern oder der Idee medialer Verantwortung nicht gerecht werden könnten. Auch die dritte Auffassung, nach der nur ein konsequenzialistischer Ansatz einen angemessenen Umgang mit den spezifischen Problemen der Medienethik ermöglicht, ist folglich unbegründet oder im besten Fall irreführend.

Freilich kann man fragen, ob die von Rath und anderen suggerierte Sonderrolle des Konsequenzialismus nicht schon durch die Realität der medienethischen Debatte widerlegt wird, liefert doch mit der Diskursethik ein nicht-konsequenzialistischer Ansatz einen der Hauptreferenzpunkte der deutschsprachigen Medienethik (vgl. auch Filipovic 2019). Obwohl die Diskursethik aber zweifelsohne zu denjenigen Theorieansätzen der normativen Ethik zählt, auf die in der medienethischen Literatur am häufigsten Bezug genommen wird, hat sie doch gerade mit Blick auf die Anwendung, d.h. die ethische Konkretisierung, ihre ganz eigenen Probleme. Eine zentrale Idee der Diskursethik besteht in der Zurückweisung monologischer Begründungsverfahren und der Auffassung, dass allein dialogisch begründete Prinzipien oder Handlungsanweisungen als gerechtfertigt betrachtet werden können und auch dies nur dann, wenn sich der betreffende Dialog unter bestimmten Bedingungen vollzieht, wie etwa dem der Herrschaftsfreiheit. Auf diese Weise wird die für jede angewandte Ethik so essenzielle ethische Konkretisierung letztlich an die Teilnehmer eines idealen Diskurses delegiert.

Die vorliegenden diskursethischen Beiträge zur Medienethik verbleiben denn auch typischerweise auf einer abstrakteren Ebene und diskutieren beispielsweise den diskursethischen Öffentlichkeitsbegriff, ohne sich eindeutig festzulegen, welche Handlungsanweisungen aus diesem Begriff abzuleiten sind (vgl. etwa Heesen 2008). Solange man daher nicht bereit ist, einige der Grundüberzeugungen des Ansatzes aufzugeben, ist von der Diskursethik eine normative und zugleich angewandte Medienethik kaum zu erwarten. 


\section{Individuelle und kollektive Verantwortung}

Der vierte und letzte „Irrtum“ setzt ebenfalls beim Begriff der Verantwortung an. Er ist aber insofern anders gelagert, als das Problem hier nur in der Voreiligkeit der Festlegung liegt, nicht darin, dass die vertretene Sichtweise nicht zu verteidigen wäre. So lautet eine weitere in der medienethischen Literatur beliebte These, dass individualethische Ansätze die Probleme der Medienethik nicht angemessen erfassen können, sondern es hierzu einer Konzeption kollektiver oder korporativer Verantwortung bedarf. Die betreffende Auffassung wird üblicherweise mit einem Hinweis auf die institutionellen Strukturen begründet, in denen sich moderne Medienakteur*innen bewegen (vgl. auch Müßigbrodt 2006, S. 142). Journalist*innen, so heißt es oft, treffen keine wirklich freie Entscheidung darüber, wie sie im Rahmen ihrer Tätigkeit verfahren und mit bestimmten moralischen Herausforderungen umgehen wollen. Stattdessen sind sie immer schon in ein komplexes Geflecht aus Hierarchien und Verhaltenserwartungen eingebunden. Aus eben diesem Grund sei eine Medienethik, die individuelle Handlungsanweisungen für Journalist*innen formulieren will, von Grund auf verfehlt.

Die Notwendigkeit einer nicht- oder über-individualistischen Perspektive ist in der Vergangenheit von einer ganzen Reihe von Medienethiker*innen betont worden (vgl. etwa Rühl und Saxer 1981, S. 482, 483 und 485; Haller und Holzhey 1992, S. 12; Teichert 1996, S. 822-23; Rath 2002, S. 64; Funiok 2002, S. 48; Krainer 2002, S. 162; Debatin 2004, S. 40-41; Saxer 2004, S. 187; Stapf 2006, S. 11; Alsdorf 2007, S. 4; Heesen 2008, S. 270-71; Hömberg und Klenk 2010, S. 42; Friedrichsen und Gertler 2011, S. 88; Karmasin und Litschka 2014, S. 372 und 374). Obwohl die meisten Autor*innen für einen vermittelnden Ansatz argumentieren, der individualistische und kollektivistische Aspekte vereint, überwiegt doch die Kritik an individualistischen Konzeptionen. Die möglichen Stärken und positiven Beiträge solcher Ansätze kommen kaum zur Sprache - eine Tatsache, die schon früh von Hermann Boventer bemängelt worden ist:

Diese nicht ganz unberechtigten Vorwürfe haben nun allerdings das Pendel ins andere Extrem ausschwingen lassen, wo die Person als journalistische Bezugseinheit ganz in den Hintergrund tritt und wo die Qualitäten des einzelnen Journalisten nur noch eine untergeordnete Rolle spielen. (Boventer 1984, S. 266)

Wie schon im Fall des Konsequenzialismus wäre eine systematische Beschränkung auf Konzeptionen kollektiver Verantwortung mit beträchtlichen Kosten verbunden. Die Optionen innerhalb des medienethischen Diskurses würden nicht nur erheblich eingeschränkt, sondern der Theoriebildung würden ernstzunehmende Lasten aufgebürdet, da sich Konzeptionen kollektiven Handelns und kollektiver Verantwortung ganz eigenen Problemen und Herausforderungen gegenüber sehen.

Auch hier zeigen diese Bedenken nicht, dass das Votum für eine Konzeption kollektiver Verantwortung der Sache nach unberechtigt ist. Ein „Irrtum“ besteht aber in der Unterstellung, eine individualethische Medienethik müsse den ,einzelnen Journalisten“ oder die ,einzelne Journalistin“ in den Mittelpunkt rücken und könne den Entscheidungen, die über seinen oder ihren Kopf hinweg getroffen werden, nicht gerecht werden. Eine individualethische Betrachtung kann auch auf anderen 
Ebenen ansetzen und das Handeln und die Entscheidungen von Herausgeber*innen, Intendant*innen, Eigentümer*innen oder auch einzelnen Mediennutzer*innen in den Blick nehmen. Gerade im Zuge der Digitalisierung und der sozialen Medien gewinnen individualethische Betrachtungen an Bedeutung, weil individuelle Nutzer*innen ganz andere Möglichkeiten der Teilhabe am öffentlichen Diskurs erlangen und oftmals in moralisch fragwürdiger Weise nutzen (vgl. auch Debatin 2017, S. 53). Aus der Tatsache, dass individuelle Journalist*innen keine freien Entscheidungen treffen und wenig Macht haben, bestehende Strukturen zu verändern, folgt daher nicht, dass eine individualethische Betrachtung verfehlt sein muss.

Dies wäre sie nur, wenn zwei weitere Bedingungen erfüllt sind: 1) Die Entscheidungen, durch die Strukturen geschaffen werden, in denen Journalist*innen und andere Medienakteur*innen agieren, werden ihrerseits nicht von mächtigen Individuen, sondern von Kollektiven getroffen. 2) Die Entscheidungen und Handlungen dieser Kollektive lassen sich nicht auf Entscheidungen und Handlungen der ihnen angehörenden Individuen reduzieren. Gerade diese zweite Annahme ist aber keineswegs trivial und erfordert eine ausführlichere philosophische Begründung, als sie in der medienethischen Literatur gemeinhin geliefert wird.

Umgekehrt liefern die oben mit Blick auf den Verantwortungsbegriff angestellten Überlegungen eine Basis, um die Notwendigkeit einer individualistischen Betrachtung zu bekräftigen (vgl. hierzu auch Stapf 2006, S. 14; Bracker 2017, 57-58). Wenn die Zuschreibung von Verantwortung letztlich in die Identifizierung konkreter Handlungsverpflichtungen münden muss, weil die Medienethik ansonsten ihrer handlungsleitenden Funktion nicht gerecht werden kann, dann kann Letztere nicht ohne eine individualethische Betrachtung auskommen. Dies heißt allerdings keineswegs, dass diese im Sinne eines tugendethischen Paradigmas umgesetzt werden müsste, wie Kritiker individualethischer Ansätze mitunter fälschlich suggerieren.

Erforderlich ist daher ein ergebnisoffener Diskurs über die Frage, welche Probleme medialen Handelns eine individualethische und welche eher eine kollektivethische Antwort erfordern, sowie eine eingehende Beschäftigung mit den jeweiligen Stärken und Schwächen der beiden Ansätze. Obwohl die bereits vorliegenden Versuche, individual- und kollektivethische Perspektiven zu integrieren und sie beispielsweise systematisch verschiedenen Analyseebenen (Mikro, Meso, Makro) zuzuweisen (vgl. etwa Karmasin und Litschka 2014; Haller 2017; Krainer 2018), Anlass zu Hoffnung geben, wird dieser Diskurs weiterhin durch die oftmals einseitige und oberflächliche Zurückweisung individualistischer Perspektiven behindert.

\section{Fazit}

Nimmt man die vier theoretischen Vorannahmen, die ich hier erörtert habe, wirklich ernst, dann sieht man sich Erwartungen gegenüber, die einem offenen und produktiven multidisziplinären Diskurs, der in konkrete Handlungsanweisungen mündet, nicht eben förderlich sind. Auch wenn ich nicht empirisch argumentiert habe, erscheint die Einschätzung plausibel, dass der insgesamt unbefriedigende Zustand der Medienethik zumindest teilweise auf diese Vorannahmen und Festlegungen zurückgeht. 
Umgekehrt kann dann aber auch gelten: Wenn die oben analysierten Irrtümer über die Möglichkeit und Form der Moralbegründung und über den Charakter von Praxisnormen überwunden werden, wenn der Verantwortungsbegriff geklärt und die Vielfalt deontologischer Ansätze anerkannt wird und wenn die Notwendigkeit von sowohl individualethischen als auch kollektivethischen Ansätzen ernst genommen und eine entsprechende Arbeitsteilung vorgenommen wird, dann ist ein offenerer medienethischer Diskurs und eine fruchtbarere wissenschaftliche Auseinandersetzung mit den ethischen Problemen und Herausforderungen medialen Handelns möglich.

Funding Open Access funding enabled and organized by Projekt DEAL.

Open Access Dieser Artikel wird unter der Creative Commons Namensnennung 4.0 International Lizenz veröffentlicht, welche die Nutzung, Vervielfältigung, Bearbeitung, Verbreitung und Wiedergabe in jeglichem Medium und Format erlaubt, sofern Sie den/die ursprünglichen Autor(en) und die Quelle ordnungsgemäß nennen, einen Link zur Creative Commons Lizenz beifügen und angeben, ob Änderungen vorgenommen wurden.

Die in diesem Artikel enthaltenen Bilder und sonstiges Drittmaterial unterliegen ebenfalls der genannten Creative Commons Lizenz, sofern sich aus der Abbildungslegende nichts anderes ergibt. Sofern das betreffende Material nicht unter der genannten Creative Commons Lizenz steht und die betreffende Handlung nicht nach gesetzlichen Vorschriften erlaubt ist, ist für die oben aufgeführten Weiterverwendungen des Materials die Einwilligung des jeweiligen Rechteinhabers einzuholen.

Weitere Details zur Lizenz entnehmen Sie bitte der Lizenzinformation auf http://creativecommons.org/ licenses/by/4.0/deed.de.

\section{Literatur}

Albertzart, M. (2015). Der Vorrang des Pflichtbegriffs in kollektiven Kontexten. Zeitschrift für Praktische Philosophie, 2, 87-120.

Alsdorf, J. (2007). Medienethik und Medienkritik. Wege zu einer politischen Philosophie der Medien. Saarbrücken: VDM.

Altendorfer, L.-M. (2019). Influencer in der digitalen Gesundheitskommunikation. Instagramer, YouTuber und Co. zwischen Qualität, Ethik und Professionalisierung. Baden-Baden: Nomos.

Altmeppen, K.-D. (2020). Vielfalt der Themen, Vielfalt der Öffentlichkeiten, Vielfalt der Verantwortung. Betrachtungen zur ethischen Mehrdimensionalität öffentlicher Kommunikation. In M. Prinzing, B. Debatin \& N. Köberer (Hrsg.), Kommunikations- und Medienethik reloaded? Wegmarken für eine Orientierungssuche im Digitalen (S. 87-104). Baden-Baden: Nomos.

Altmeppen, K.-D., Bieber, C., Filipovic, A., Heesen, J., Neuberger, C., Röttger, U., Stiegler, S., \& Thomas, T. (2019). Öffentlichkeit, Verantwortung und Gemeinwohl im digitalen Zeitalter. Publizistik, 64, 59-74.

Altmeppen, K.-D., Büsch, A., \& Filipovic, A. (2013). Medienethik als Aufgabe und Verpflichtung. Zur Neuausrichtung von Communicatio Socialis. Communicatio Socialis, 46, 280-287.

Bartholomew, J. (2015). Easy virtue. The Spectator. https://www.spectator.co.uk/article/easy-virtue. Zugegriffen: 26. Mai 2021.

Bayertz, K. (1995). Eine kurze Geschichte der Herkunft der Verantwortung. In K. Bayertz (Hrsg.), Verantwortung - Prinzip oder Problem? (S. 3-71). Darmstadt: Wissenschaftliche Buchgesellschaft.

Bayertz, K. (2008). Was ist Angewandte Ethik? In J. S. Ach, K. Bayertz \& L. Siep (Hrsg.), Grundlagen. Grundkurs Ethik, (Bd. 1, S. 165-179). Paderborn: Mentis.

Bayertz, K., \& Beck, B. (2017). Der Begriff der Verantwortung in der Moderne: 19.-20. Jahrhundert. In L. Heidbrink, C. Langbehn \& J. Loh (Hrsg.), Handbuch Verantwortung (S. 133-147). Wiesbaden: Springer VS. 
Beauchamp, T.L., \& Childress, J.F. (2019). Principles of biomedical ethics (8. Aufl.). New York: Oxford University Press.

Bechthold-Hengelhaupt, T. (2021). Fake News und Desinformation aus der Sicht der Theorie sozialer Systeme. In C. Schicha, I. Stapf \& S. Sell (Hrsg.), Medien und Wahrheit. Medienethische Perspektiven auf Desinformation, Lügen und „Fake News“ (S. 135-151). Baden-Baden: Nomos.

Betz, A., Giebeler, M., Gutscher, M., Inman, X., Nagel, M., Rothfuß, D., Schäfer, J.H., \& Zöllner, O. (2018). Macht Porno glücklich? Eine empirische Studie zu Nutzung und Ethik von Pornografie im Internet. Köln: Bundesanzeiger Verlag.

Birnbacher, D. (1995). Grenzen der Verantwortung. In K. Bayertz (Hrsg.), Verantwortung - Prinzip oder Problem? (S. 143-183). Darmstadt: Wissenschaftliche Buchgesellschaft.

Black, J., \& Roberts, C. (2011). Doing ethics in media: theories and practical applications. New York: Routledge.

Bobbitt, R. (2020). Exploring communication ethics. A socratic approach. New York: Routledge.

Boventer, H. (1984). Ethik des Journalismus. Zur Philosophie der Medienkultur. Konstanz: Universitätsverlag.

Bracker, I. (2017). Verantwortung von Medienunternehmen. Selbstbild und Fremdwahrnehmung in der öffentlichen Kommunikation. Baden-Baden: Nomos.

Brosda, C., \& Schicha, C. (2010). Einleitung. In C. Brosda \& C. Schicha (Hrsg.), Handbuch Medienethik (S. 9-17). Wiesbaden: VS.

Christians, C. G. (2013). Global ethics and the problem of relativism. In S. J. A. Ward (Hrsg.), Global media ethics. Problems and perspectives (S. 272-294). Chichester: Wiley-Blackwell.

Christians, C. G. (2019). Media ethics and global justice in a digital age. Cambridge: Cambridge University Press.

Cooper, T.W., \& Christians, C.G. (2020). The search for universals. In L. Wilkins \& C. G. Christians (Hrsg.), The Routledge handbook of mass media ethics (S. 73-87). New York: Routledge.

Couldry, N. (2013). Why media ethics still matters. In S. J. A. Ward (Hrsg.), Global media ethics. Problems and perspectives (S. 13-29). Chichester: Wiley-Blackwell.

Debatin, B. (2004). Medienethik als Steuerungsinstrument? Zum Verhältnis von individueller und korporativer Verantwortung in der Medienethik. In A. Holderegger (Hrsg.), Kommunikations- und Medienethik. Interdisziplinäre Perspektiven (S. 39-54). Fribourg: Academic Press.

Debatin, B., \& Funiok, R. (2003). Begründungen und Argumentationen der Medienethik - ein Überblick. In B. Debatin \& R. Funiok (Hrsg.), Kommunikations- und Medienethik (S. 9-20). Konstanz: UVK.

Debatin, B. (2015). Das Ende der journalistischen Ethik? Gedanken zur Verortung des Journalismus im 21. Jahrhundert am Beispiel der USA und zur Neubestimmung seiner ethischen Aufgabe. In M. Prinzing, M. Rath, C. Schicha, \& I. Stapf (Hrsg.), Neuvermessung der Medienethik. Bilanz, Themen und Herausforderungen seit 2000 (S. 56-73). Weinheim: Beltz Juventa.

Debatin, B. (2017). Wandel des Journalismus, Erweiterung der Journalismus- und Medienethik? In I. Stapf, M. Prinzing, \& A. Filipovic (Hrsg.), Gesellschaft ohne Diskurs? Digitaler Wandel und Journalismus aus medienethischer Perspektive (S. 53-67). Baden-Baden: Nomos.

Dernbach, B. (2017). Narration und Storytelling im medienethischen Diskurs. In I. Stapf, M. Prinzing \& A. Filipovic (Hrsg.), Gesellschaft ohne Diskurs? Digitaler Wandel und Journalismus aus medienethischer Perspektive (S. 151-164). Baden-Baden: Nomos.

Eggers, D. (2019). Kontrolle ist besser. In W. Hinsch \& D. Eggers (Hrsg.), Öffentliche Vernunft? Die Wissenschaft in der Demokratie (S. 61-72). Berlin:: de Gruyter.

Filipovic, A. (2015). Angewandte Ethik. Grundbegriffe der Kommunikations- und Medienethik, Teil 2. Communicatio Socialis, 48, 431-437.

Filipovic, A. (2016). Angewandte Ethik. In J. Heesen (Hrsg.), Handbuch Medien- und Informationsethik (S. 41-51). Stuttgart: Metzler.

Filipovic, A. (2019). Alles Habermas!? Alternative Theorien für eine Ethik öffentlicher Kommunikation in Zeiten der Digitalität. In J. Bedford-Strohm, F. Höhne \& J. Zeyher-Quattlender (Hrsg.), Digitaler Strukturwandel der Öffentlichkeit (S. 219-232). Baden-Baden: Nomos.

Filipovic, A., Schicha, C., \& Stapf, I. (2017). Vorwort der Reihenherausgeber. In I. Bracker, Verantwortung von Medienunternehmen. Selbstbild und Fremdwahrnehmung in der öffentlichen Kommunikation (S. 3-4). Baden-Baden: Nomos.

Fox, C., \& Saunders, J. (Hrsg.). (2019). Media ethics, free speech, and the requirements of democracy. New York: Routledge.

Friedrichsen, M., \& Gertler, M. (2011). Medien zwischen Ökonomie und Qualität. Medienethik als Instrument der Medienwirtschaft. Baden-Baden: Nomos.

Frost, C. (2016). Journalism ethics and regulation. Abingdon: Routledge. 
Funiok, R. (2002). Medienethik. In M. Karmasin (Hrsg.), Medien und Ethik (S. 37-58). Stuttgart: Reclam. Funiok, R. (2011). Medienethik. Verantwortung in der Mediengesellschaft. Stuttgart: Kohlhammer.

Funiok, R. (2015). Hauptthemen und Autoren in der Entwicklung der deutschsprachigen Kommunikations- und Medienethik. In M. Prinzing, M. Rath, C. Schicha \& I. Stapf (Hrsg.), Neuvermessung der Medienethik. Bilanz, Themen und Herausforderungen. seit, (Bd. 2000, S. 20-34). Weinheim: Beltz Juventa.

Gathmann, F. (2020). Wir müssen so neutral sein wie möglich. Der Spiegel. https://www.spiegel.de/kultur/ wir-muessen-so-neutral-sein-wie-moeglich-a-1820e9d1-9cf6-4d3f-8965-aa7a59f8ec66. Zugegriffen: 26. Juni 2020.

Gossel, B. M. (2018). Entrepreneurial Journalism. Chance für die journalistische Freiheit? Reflexion des Freiheitsbegriffs und Konsequenzen für die Journalistenausbildung. In A. Czepek, M. Hellwig, B. Illg, \& E. Nowak (Hrsg.), Freiheit und Journalismus (S. 79-96). Baden-Baden: Nomos.

Greis, A. (2003). Die immanenten Strukturen medialer Kommunikation als Fokus medienethischer Anstrengungen. Eine Methodologie. In A. Greis, G. W. Hunold \& K. Koziol (Hrsg.), Medienethik. Ein Arbeitsbuch (S. 3-18). Tübingen: Francke.

Greis, A., Hunold, G. W., \& Koziol, K. (2003). Vorwort. In A. Greis, G. W. Hunold \& K. Koziol (Hrsg.), Medienethik. Ein Arbeitsbuch (S. ix-xiv). Tübingen: Francke.

Haller, M. (2017). Die digitalen Medien: Rückkehr zur Individualmoral? Über die Lehrbarkeit ethisch gerechtfertigten Handelns im Web. In I. Stapf, M. Prinzing \& A. Filipovic (Hrsg.), Gesellschaft ohne Diskurs? Digitaler Wandel und Journalismus aus medienethischer Perspektive (S. 17-33). BadenBaden: Nomos.

Haller, M., \& Holzhey, H. (1992). Einleitung: Die Frage nach einer Medienethik. In M. Haller \& H. Holzhey (Hrsg.), Medien-Ethik (S. 11-19). Opladen: Westdeutscher Verlag.

Hanitzsch, T., Plaisance, P. L., \& Skewes, E. A. (2013). differences, 30(49), 30-49.

Heesen, J. (2008). Medienethik und Netzkommunikation. Öffentlichkeit in der individualisierten Mediengesellschaft. Frankfurt/M.: Humanities Online.

Hinsch, W. (2017). Die Moral des Krieges. München: Piper.

Hömberg, W., \& Klenk, C. (2010). Individualethische Ansätze. In C. Brosda \& C. Schicha (Hrsg.), Handbuch Medienethik (S. 41-52). Wiesbaden: VS.

Jackob, N. (2018). Die Mediengesellschaft und ihre Opfer. Grenzfälle journalistischer Ethik im frühen einundzwanzigsten Jahrhundert. Berlin:: Peter Lang.

Kaiser, M., \& Zeilinger, T. (2021). Ethische Standards für Roboterjournalismus? Ergebnisse und Implikationen einer Feldstudie. In C. Schicha, I. Stapf \& S. Sell (Hrsg.), Medien und Wahrheit. Medienethische Perspektiven auf Desinformation, Lügen und „Fake News“ (S. 299-316). Baden-Baden: Nomos.

Karmasin, M., \& Litschka, M. (2014). Medienethik als Wirtschaftsethik medialer Kommunikation? Möglichkeiten und Grenzen der Integration zweier aktueller Bereichsethiken. In M. Maring (Hrsg.), Bereichsethiken im interdisziplinären Dialog (S. 367-382). Karlsruhe: KIT Scientific Publishing.

Keel, G. (2018). Grenzen der Satire in der täglichen Arbeit von Karikaturisten. In A. Czepek, M. Hellwig, B. Illg \& E. Nowak (Hrsg.), Freiheit und Journalismus (S. 97-108). Baden-Baden: Nomos.

Keel, G., Dingerkus, P., \& Wyss, V. (2018). Journalistische Autonomie und Qualitätsmanagement - ein Widerspruch? In A. Czepek, M. Hellwig, B. Illg \& E. Nowak (Hrsg.), Freiheit und Journalismus (S. 69-78). Baden-Baden: Nomos.

Kelm, O., Dohle, M., \& Ryba, N. (2021). Diese „Fake News“ kommen doch von den Anderen! Wahrnehmungen und Beurteilungen von Desinformationen innerhalb divergenter Meinungslager in der Flüchtlingsdebatte. In C. Schicha, I. Stapf \& S. Sell (Hrsg.), Medien und Wahrheit. Medienethische Perspektiven auf Desinformation, Lügen und „Fake News“ (S. 223-239). Baden-Baden: Nomos.

Klaidman, S., \& Beauchamp, T.L. (1987). The virtuous journalist. New York: Oxford University Press.

Köberer, N. (2015). Medienethik als angewandte Ethik. Eine wissenschaftssystematische Verortung. In M. Prinzing, M. Rath, C. Schicha \& I. Stapf (Hrsg.), Neuvermessung der Medienethik. Bilanz, Themen und Herausforderungen. seit, (Bd. 2000, S. 99-113). Weinheim: Beltz Juventa.

Koinig, I., Voci, D., Weder, F., \& Karmasin, M. (2019). Media social responsibility an der Schnittstelle von media accountability and corporate social responsibility. In M. Litschka \& L. Krainer (Hrsg.), Der Mensch im digitalen Zeitalter. Zum Zusammenhang von Ökonomisierung, Digitalisierung und Mediatisierung (S. 103-134). Wiesbaden: Springer VS.

Koska, C. (2015). Zur Idee einer digitalen Bildungsidentität. In H. Gapski (Hrsg.), Big Data und Medienbildung. Zwischen Kontrollverlust, Selbstverteidigung und Souveränität in der digitalen Welt (S. 81-94). Düsseldorf: Kopaed. 
Krainer, L. (2002). Medienethik als angewandte Ethik. Zur Organisation ethischer Entscheidungsprozesse. In M. Karmasin (Hrsg.), Medien und Ethik (S. 156-174). Stuttgart: Reclam.

Krainer, L. (2015). Medienethik als Aufgabe inter- und transdisziplinärer Reflexionsleistung. Ein Beitrag zur deutschsprachigen Fachgeschichte und Fachzukunft. In M. Prinzing, M. Rath, C. Schicha \& I. Stapf (Hrsg.), Neuvermessung der Medienethik. Bilanz, Themen und Herausforderungen seit 2000 (S. 35-55). Weinheim: Beltz Juventa.

Krainer, L. (2018). Kollektive Autonomie als kommunikations- und medienethische Selbstbestimmung. Medien \& Kommunikationswissenschaft, 66, 485-501.

Krainer, L., \& Karmasin, M. (2020). Bewegte Objekte? Erweiterung medienethischer Objektbereiche und Konsequenzen für das Fach der Kommunikations- und Medienethik. In M. Prinzing, B. Debatin \& N. Köberer (Hrsg.), Kommunikations- und Medienethik reloaded? Wegmarken für eine Orientierungssuche im Digitalen (S. 309-322). Baden-Baden: Nomos.

Krämer, C. (2020). Menschenwürde und Reality TV. Ein Widerspruch? Baden-Baden: Nomos.

Lauerer, C. (2018). Bröckelt die Brandschutzmauer? Werbeinteressen und ihr Einfluss auf journalistische Arbeit in Medienunternehmen. In A. Czepek, M. Hellwig, B. Illg \& E. Nowak (Hrsg.), Freiheit und Journalismus (S. 123-140). Baden-Baden: Nomos.

Leif, T. (2018). Das Schattenmanagement des Journalismus. Wie „Strategische Kommunikationssteuerung" von Lobbyisten und Agenturen die journalistische Unabhängigkeit angreift. In K. Liesem \& L. Rademacher (Hrsg.), Die Macht der strategischen Kommunikation. Medienethische Perspektiven der Digitalisierung (S. 33-54). Baden-Baden: Nomos.

Leschke, R. (2001). Einführung in die Medienethik. München: Fink.

Levy, N. (2020). Virtue signalling is virtuous. Synthese. https://doi.org/10.1007/s11229-020-02653-9.

Liesem, K. (2018). Freiheit und Journalismus. Die Verdachtsberichterstattung im Spannungsverhältnis zwischen Pressefreiheit und Persönlichkeitsschutz. In A. Czepek, M. Hellwig, B. Illg \& E. Nowak (Hrsg.), Freiheit und Journalismus (S. 109-121). Baden-Baden: Nomos.

Luce, A. (Hrsg.). (2019). Ethical reporting of sensitive topics. London: Routledge.

Luhmann, N. (2008a). Normen in soziologischer Perspektive. In N. Luhmann (Hrsg.), Die Moral der Gesellschaft (S. 25-55). Frankfurt a. M.: Suhrkamp.

Luhmann, N. (2008b). Ethik als Reflexionstheorie der Moral. In N. Luhmann, Die Moral der Gesellschaft (S. 270-347). Frankfurt a. M.: Suhrkamp.

Maisel, L.S., \& Dineen, H.E. (Hrsg.). (2018). Trumping ethical norms. Teachers, preachers, pollsters, and the media respond to Donald Trump. New York: Routledge.

Maring, M. (2014). Einleitung und Übersicht. In M. Maring (Hrsg.), Bereichsethiken im interdisziplinären Dialog (S. 9-23). Karlsruhe: KIT Scientific Publishing.

Medvecky, F., \& Leach, J. (2019). An ethics of science communication. Cham: Palgrave.

Metz, T. (2018). Cultural pluralism and media ethics: Theorizing in a globalized world of difference. In P. L. Plaisance (Hrsg.), Communication and media ethics (S. 53-73). Boston: De Gruyter.

Mikos, L. (2010). Real life Formate. In C. Brosda \& C. Schicha (Hrsg.), Handbuch Medienethik (S. 431-441). Wiesbaden: VS.

Müller, U., Feiks, M., Krautter, J., \& Zurstiege, G. (2018). Ethik der Werbung in Zeiten der Digitalisierung. In K. Liesem \& L. Rademacher (Hrsg.), Die Macht der strategischen Kommunikation. Medienethische Perspektiven der Digitalisierung (S. 141-157). Baden-Baden: Nomos.

Müßigbrodt, K. (2006). Journalismus und der Schutz des Privaten. Paderborn: Mentis.

Nikolaev, A. G. (2018). Searching for universals without making problematic imperialistic assumptions. In P.L. Plaisance (Hrsg.), Communication and media ethics (S. 529-548). Boston: De Gruyter.

Oehmke, P. (2020). Die Zeit der Neutralität ist vorbei. Der Spiegel. https://www.spiegel.de/kultur/newyork-times-die-zeit-der-neutralitaet-ist-vorbei-a-5ccaa4e4-eca2-4a2e-b2d7-22e6a484f8ce. Zugegriffen: 26. Juni 2020.

Paganini, C. (2018). Entwurf einer rekonstruktiven Medienethik. Analyse und Auswertung internationaler und nationaler Selbstverpflichtungskodizes. München: zem::dg.

Paganini, C. (2020). Werte für die Medien(ethik). Baden-Baden: Nomos.

Porlezza, C. (2020). Ethische Herausforderungen des automatisierten Journalismus. Zwischen Dataismus, Bias und fehlender Transparenz. In M. Prinzing, B. Debatin \& N. Köberer (Hrsg.), Kommunikations- und Medienethik reloaded? Wegmarken für eine Orientierungssuche im Digitalen (S. 143-158). Baden-Baden: Nomos.

Prinzing, M., Rath, M., Schicha, C., \& Stapf, I. (2015). Einleitung: Neuvermessung der Medienethik. In M. Prinzing, M. Rath, C. Schicha \& I. Stapf (Hrsg.), Neuvermessung der Medienethik. Bilanz, Themen und Herausforderungen seit 2000 (S. 9-18). Weinheim: Beltz Juventa. 
Rath, M. (2002). Medienqualität zwischen Empirie und Ethik. In M. Karmasin (Hrsg.), Medien und Ethik (S. 59-76). Stuttgart: Reclam.

Rath, M. (2010). Empirische Perspektiven. In C. Brosda \& C. Schicha (Hrsg.), Handbuch Medienethik (S. 136-146). Wiesbaden: VS.

Rawls, J. (1971). A theory of justice. Cambridge: The Belknap Press.

Ring, S., \& Funiok, R. (2015). Harmloses Als-Ob, nützliches Lebenstraining, problematische Menschenbilder - Braucht es eigene ethische Maximen für Computerspiele? In M. Prinzing, M. Rath, C. Schicha \& I. Stapf (Hrsg.), Neuvermessung der Medienethik. Bilanz, Themen und Herausforderungen seit 2000 (S. 177-190). Weinheim: Beltz Juventa.

Rühl, M., \& Saxer, U. (1981). 25 Jahre Deutscher Presserat. Ein Anlaß für Überlegungen zu einer kommunikationswissenschaftlich fundierten Ethik des Journalismus und der Massenkommunikation. $\mathrm{Pu}$ blizistik, 26, 471-507.

Saxer, U. (2004). Journalistische Ethik im elektronischen Zeitalter - eine Chimäre? In A. Holderegger (Hrsg.), Kommunikations- und Medienethik. Interdisziplinäre Perspektiven (S. 184-197). Fribourg: Academic Press.

Schicha, C. (2010a). Medienskandale. In C. Brosda \& C. Schicha (Hrsg.), Handbuch Medienethik (S. 373-330). Wiesbaden: VS.

Schicha, C. (2010b). Philosophische Ethik. In C. Brosda \& C. Schicha (Hrsg.), Handbuch Medienethik (S. 21-40). Wiesbaden: VS.

Schicha, C. (2021). Bearbeitete Bilder - Techniken und Bewertungen visueller Veränderungen am Beispiel politischer Motive. In C. Schicha, I. Stapf \& S. Sell (Hrsg.), Medien und Wahrheit. Medienethische Perspektiven auf Desinformation, Lügen und "Fake News" (S. 173-204). Baden-Baden: Nomos.

Schöne-Seifert, B. (2011). Prinzipien und Theorien der Medizinethik. In J. S. Ach, K. Bayertz \& L. Siep (Hrsg.), Anwendungen. Grundkurs Ethik, (Bd. 2, S. 9-21). Paderborn: Mentis.

Sell, S., \& Oswald, B. (2021). Verifikation von Online-Inhalten im Journalismus. In C. Schicha, I. Stapf \& S. Sell (Hrsg.), Medien und Wahrheit. Medienethische Perspektiven auf Desinformation, Lügen und „Fake News" (S. 243-262). Baden-Baden: Nomos.

Stapf, I. (2006). Medien-Selbstkontrolle. Ethik und Institutionalisierung. Konstanz: UVK.

Stapf, I. (2020). Was sagen uns „Fake News“ (nicht) über Wahrheit? Medienethische Überlegungen zu Wahrheit und Wahrhaftigkeit im digitalen Zeitalter. In M. Prinzing, B. Debatin \& N. Köberer (Hrsg.), Kommunikations- und Medienethik reloaded? Wegmarken für eine Orientierungssuche im Digitalen (S. 231-250). Baden-Baden: Nomos.

Teichert, W. (1996). Medienethik. In J. Nida-Rümelin (Hrsg.), Angewandte Ethik (S. 805-833). Stuttgart: Kröner.

Thies, C. (2011). Medienethik. In R. Stoecker, C. Neuhäuser \& M.-L. Raters (Hrsg.), Handbuch Angewandte Ethik (S. 206-209). Stuttgart: Metzler.

Ward, S. J. A. (2011). Ethics and the media. An introduction. Cambridge: Cambridge University Press.

Ward, S. J. A. (2015). Radical media ethics. A global approach. Chichester: Wiley-Blackwell.

Weber, K. (2016). Welche Gerechtigkeit kann gendersensible Gerechtigkeit in der Mediendebatte sein? Zur Unübersichtlichkeit einer kaum geführten Debatte. In S. Kannengießer, L. Krainer, C. Riesmeyer \& I. Stapf (Hrsg.), Eine Frage der Ethik? Eine Ethik des Fragens. Interdisziplinäre Untersuchungen zu Medien, Ethik und Geschlecht (S. 48-63). Weinheim: Beltz Juventa.

Weischenberg, S. (2018). Medienkrise und Medienkrieg. Brauchen wir überhaupt noch Journalismus? Wiesbaden: Springer.

Werner, M. H. (2020). Minimalgehalte und Grenzen der Verantwortungszuschreibung. In A. Seibert-Fohr (Hrsg.), Entgrenzte Verantwortung. Zur Reichweite und Regulierung von Verantwortung in Wirtschaft, Medien, Technik und Umwelt (S. 31-48). Berlin: Springer.

Wessler, H., Haffner, P., \& Rinke, E. M. (2018). Selbstbestimmung in der digitalen Welt. Über die Vorteile eines ebenenübergreifenden normativen Basiskonzepts für die empirische Erforschung der digitalen Kommunikation. Medien \& Kommunikationswissenschaft, 66, 395-406.

Wiedel, F. (2019). Die Grenzen der Aufmerksamkeit: Mentale Überlastungen in einer mediatisierten Gesellschaft. In M. Litschka \& L. Krainer (Hrsg.), Der Mensch im digitalen Zeitalter. Zum Zusammenhang von Ökonomisierung, Digitalisierung und Mediatisierung (S. 49-86). Wiesbaden: Springer VS.

Wiegerling, K. (1998). Medienethik. Stuttgart: Metzler.

Wladarsch, J. (2019). Metakommunikation und die Qualität des Journalismus. Einfluss von Metakommunikation auf Qualitätserwartungen und -bewertungen bei Nachrichtennutzern im Internet. BadenBaden: Nomos. 
Wunden, W. (1999). Freiheitliche Medienmoral. Konzept einer systematischen Medienethik. In R. Funiok, U. F. Schmölzle \& C. H. Werth (Hrsg.), Medienethik - die Frage der Verantwortung (S. 35-55). Bonn: Bundeszentrale für politische Bildung.

Zurstiege, G. (2018). Werbeethik als angewandte Ethik. Ein Plädoyer für eine Ethik, die sich einmischt. In K. Liesem \& L. Rademacher (Hrsg.), Die Macht der strategischen Kommunikation. Medienethische Perspektiven der Digitalisierung (S. 129-139). Baden-Baden: Nomos.

Prof. Dr. Daniel Eggers ist Professor für Geschichte der Philosophie an der Universität Regensburg. 